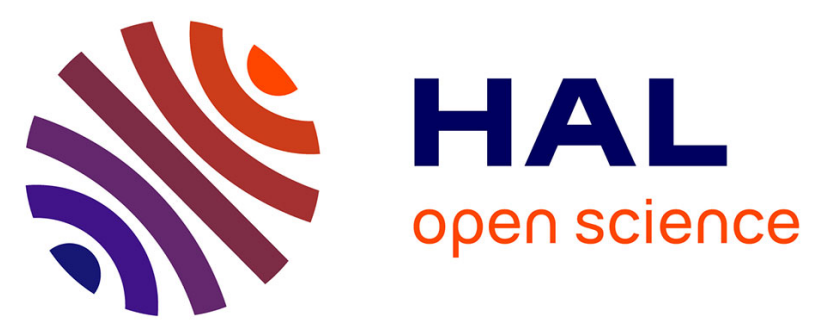

\title{
Les Delphiens au miroir de leurs offrandes monumentales : élite sociale et notabilité politique dans une petite cité de Grèce centrale (IVe-Ier s. av. J.-C.)
}

Nicolas Kyriakidis

\section{- To cite this version:}

Nicolas Kyriakidis. Les Delphiens au miroir de leurs offrandes monumentales : élite sociale et notabilité politique dans une petite cité de Grèce centrale (IVe-Ier s. av. J.-C.). Bulletin de Correspondance Hellenique, 2014, 138.1, pp.103-129. hal-02558932

HAL Id: hal-02558932

https://hal-univ-paris8.archives-ouvertes.fr/hal-02558932

Submitted on 19 Jan 2021

HAL is a multi-disciplinary open access archive for the deposit and dissemination of scientific research documents, whether they are published or not. The documents may come from teaching and research institutions in France or abroad, or from public or private research centers.
L'archive ouverte pluridisciplinaire HAL, est destinée au dépôt et à la diffusion de documents scientifiques de niveau recherche, publiés ou non, émanant des établissements d'enseignement et de recherche français ou étrangers, des laboratoires publics ou privés. 


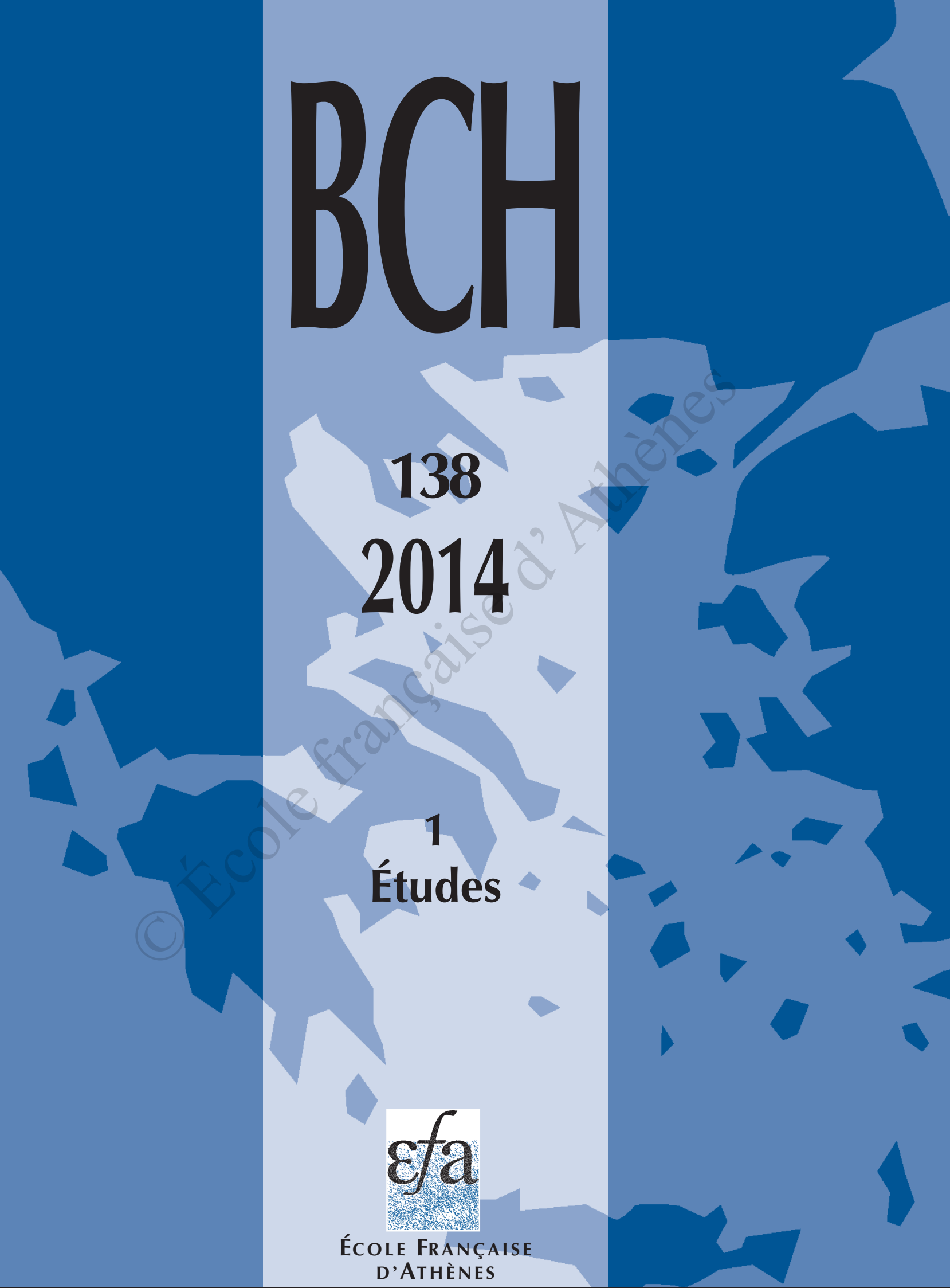

BULLETIN DE CORRESPONDANCE HELLÉNIQUE 
É C O L E F R A N ÇA I S E D'A T H È N E S
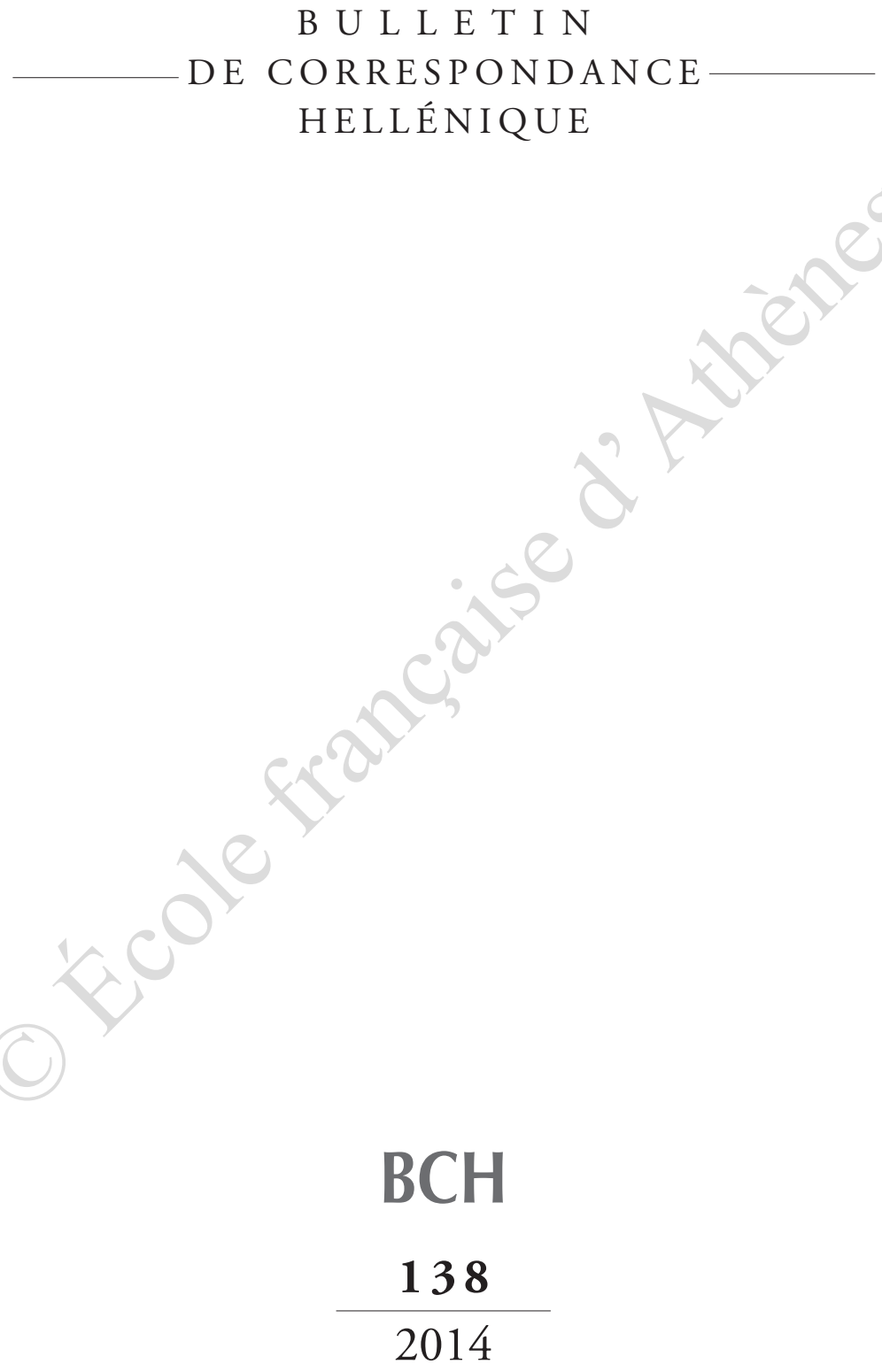
É C O L E F R A N ÇA I S E D'A T H È N E S

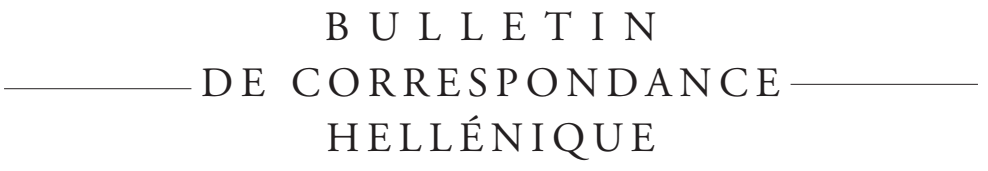

Études

$\mathrm{BCH}$

138

2014 


\section{É C O L E F R A N Ç A I S E D'A T H È N E S}

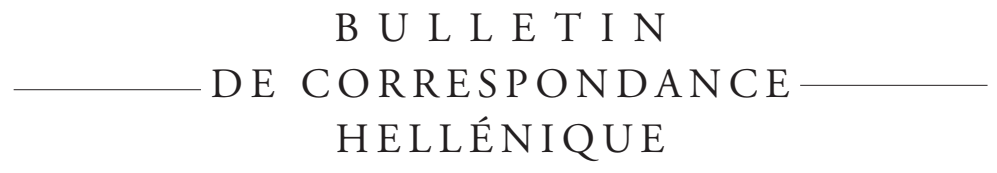

138.12014

Comité de rédaction : Alexandre FARnoux, directeur

Géraldine HuE, responsable des publications

\section{COMITÉ DE LECTURE}

Le comité de lecture de l'École française d'Athènes est composé de trois membres de droit et de neuf membres désignés par le conseil scientifique sur proposition du directeur. Sa composition actuelle est la suivante (conseil scientifique de l'École française d'Athènes du 25 juin 2012) :
Membres $\mid$ - le directeur de l'École française d'Athènes: Alexandre Farnoux
de droit $\quad$ - le directeur des études antiques et byzantines : Julien Fournier
- le directeur des études modernes et contemporaines : Maria Couroucli

Membres $\quad$ Sont membres désignés des personnalités scientifiques françaises ou étrangères (mais francophones), reconnues et désignés de dimension internationale. Le choix en est fait de manière à assurer la meilleure représentation possible des champs disciplinaires concernés. Leur mandat coïncide avec la durée d'un contrat quinquennal.

- Polixeni Adam-Veleni, Directrice du musée archéologique de Thessalonique

- Olivier Deslondes, Professeur des Universités, Université Lyon 2-Lumière

- Emanuele Greco, Directeur de l'École italienne d'Athènes

- Jean Guilaine, Professeur au Collège de France

- Miltiade B. Hatzopoulos, Directeur de recherche, Directeur du Centre de recherche sur l'Antiquité gréco-romaine (Fondation nationale de la recherche [EIE] - Athènes)

- Catherine Morgan, Directrice de l'École britannique d'Athènes

- Kosmas Pavlopoulos, Professeur à l'Université Harokopio d'Athènes

- Jean-Pierre Sodini, Professeur émérite de l'université Paris 1 - Panthéon-Sorbonne

- Georges Tolias, Directeur de recherche en histoire contemporaine, Institut de recherche néohellénique (Fondation nationale de la recherche [EIE] - Athènes)

Le comité de lecture fait appel en tant que de besoin à des experts extérieurs.

Révision des textes : EFA, Sophie Duthion

Traductions en grec : Pavlos Karvonis

Traductions en anglais : Michael WEDDE

Réalisation en PAO : EFA, Guillaume Fuchs

Impression et reliure : n.v. PEETERS s.a.

(C) École française d'Athènes, 2015

6, rue Didotou GR - 10680 Athènes www.efa.gr

Dépositaire : de Boccard Édition-Diffusion 11, rue de Médicis F - 75006 Paris www. deboccard.com

ISBN 978-2-86958-270-5

ISSN 0007-4217

Reproduction et traduction, même partielles, interdites sans l'autorisation de l'éditeur pour tous pays, y compris les États-Unis. 


\section{AVIS AUX LECTEURS}

\section{Chronique en ligne}

Partageant une longue tradition, l'École française d'Athènes et la British School at Athens diffusent auprès de la communauté scientifique le résultat de l'activité archéologique conduite en Grèce et dans certaines régions du monde hellénique. Depuis 1920, l'École française d'Athènes consacre une partie du Bulletin de correspondance hellénique à la chronique des travaux archéologiques réalisés en Grèce, à Chypre et, selon un rythme bisannuel, dans le Bosphore cimmérien. De son côté, la British School at Athens compile un bilan annuel similaire, Archaeology in Greece, publié en association avec la Society for the Promotion of Hellenic Studies comme partie constitutive des Archaeological Reports depuis 1955. Chacune des deux institutions avait un double défi à relever : faire face à une documentation croissante, d'une part; utiliser des outils plus performants pour mieux faire circuler l'information scientifique et en permettre une meilleure utilisation, d'autre part. - L'École britannique a accepté sans hésitation le projet d'un programme commun que lui a proposé l'École française d'Athènes et les deux institutions ont décidé d'unir leurs efforts, pour proposer depuis la fin de l'année 2009 une Chronique des fouilles en ligne consultable sur http://chronique.efa.gr.

Outre les articles relatifs à des opérations de terrain ou relevant de l'archéométrie, le second fascicule du $B C H$ ne comprend donc plus désormais que les "Rapports sur les travaux de l'École française d'Athènes " proposés par les responsables de missions ou de programmes.

\section{AVIS AUX AUTEURS}

Depuis la parution du $B C H 130$ (2006), les tirages à part sont fournis aux auteurs sous format électronique et sont uniquement destinés à une utilisation privée. L'École française d'Athènes conserve le copyright sur les articles, qui ne peuvent donc être mis en accès libre sur quelque base de données ou par quelque portail que ce soit. - L'ensemble de la livraison sera disponible sur le portail Persée trois ans après sa parution (www.persee.fr). 


\section{SOMMAIRE DE LA LIVRAISON}

\section{Bernard Holtzmann}

IG I3 4 : l'Acropole en chantier.

Andrzej S. Chankowski

L'éphébie athénienne antérieure à la réforme d'Epikratès : à propos de Reinmuth,

Eph.Inscr. 1 et de la chronologie des premières inscriptions éphébiques.

Julien Fournier

Retour sur un décret thasien : la donation testamentaire de Rebilus.

Nicolas Kyriakidis

Les Delphiens au miroir de leurs offrandes monumentales : élite sociale

et notabilité politique dans une petite cité de Grèce centrale (IVe-Ier s. av. J.-C.)

Jean-François BommelaER

Delphica 5. Une niche étolienne en bas du sanctuaire d'Apollon?

Jean-François Bommelaer

Delphica 6. Note sur l'escalier du théâtre de Delphes (SD 541)

Lucia A. Scatozza Höricht

Dono e reciprocità: anathemata frigi e lidi in santuari greci.

Clarisse PrÊTre, Philippe CharLIER

Stigmata et grammata dans les récits de guérisons miraculeuses d'Épidaure.

Une nouvelle analyse sémantique et clinique

Hélène Brun, Alain DuplouY

Le sanctuaire de Déméter à Vamiès (Itanos, Crète orientale). Topographie, architecture et petite plastique de terre cuite. $201-244$

Sidonie LeJEune

Le sanctuaire de Kafizin, nouvelles perspectives. $245-327$

Élise MORERo

Les techniques de fabrication de la vaisselle de pierre de Myrtos-Pyrgos $329-360$ 
Anna Georgiadou

Productions et styles régionaux dans l'artisanat céramique

de Chypre à l'époque géométrique (XIe-VIII s. av. J.-C.)

Pierre Dupont, Alexandre Baralis

Ateliers céramiques et réseaux d'échanges dans le Sud-Ouest de la mer Noire à l'époque classique et au début de l'époque hellénistique.

Nikos Akamatis

Pelops on an Early Fourth Century BC Krater from Pella

$429-448$ 


\title{
Les Delphiens au miroir de leurs offrandes monumentales
}

\author{
Élite sociale et notabilité politique dans une petite cité de \\ Grèce centrale (IV $\mathrm{I}^{\mathrm{e}}{ }^{\mathrm{er}} \mathrm{s}$. av. J.-C.)
}

\author{
Nicolas KYriakidis
}

Résumé Létude de la trentaine de monuments dédiés par des Delphiens permet d'en esquisser une lecture sociale et politique et de conclure que la société delphique a connu deux régimes successifs de l'offrande monumentale. Lors d'une première période ( $\mathrm{IV}^{\mathrm{e}}-\mathrm{III}^{\mathrm{e}} \mathrm{s}$. av. J.-C.), il ne semble pas y avoir de forte corrélation entre dédicace d'offrandes monumentales et exercice du pouvoir politique dans la cité. Au début du II ${ }^{e}$ s., alors même que la nature démocratique des institutions n’est plus contestable, le sommet de la notabilité politique de Delphes décide de se mettre en scène à travers des monuments qui exaltent l'unité de la famille et l'excellence de ses membres. Le mouvement de concentration de l'exercice du pouvoir politique dans un milieu étroit lors de la seconde moitié du Ires. av. J.-C. a pu s'appuyer sur des pratiques de distinction sociale déjà courantes depuis plus d'un siècle.

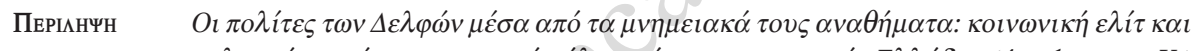

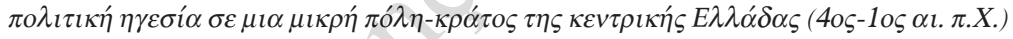

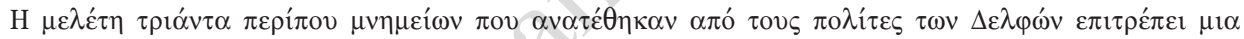

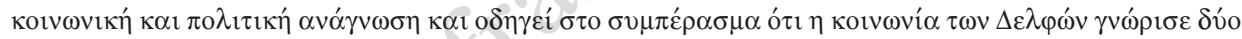

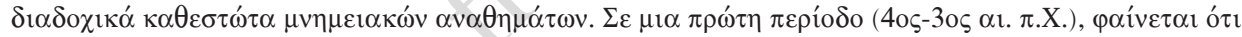

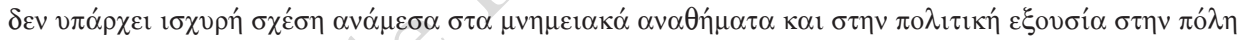

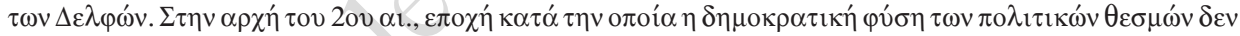

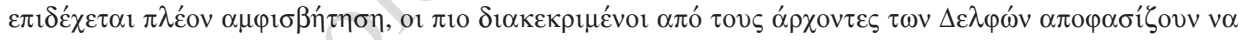

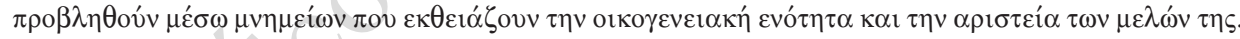

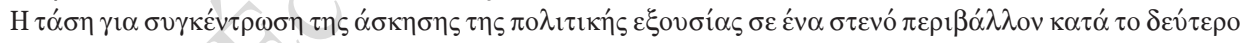

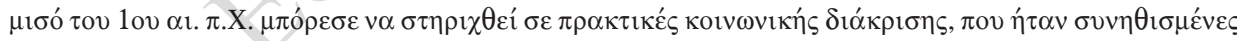

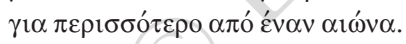

Summary The Delphians in the Mirror of their Monumental Offerings: Social Elite and Political Notability in a Small City-State of Central Greece (4th-1st century BC)

The study of about thirty monuments dedicated by Delphians allows to outline a social and political reading and to conclude that Delphic society experienced two successive regimes of monumental offering. During a first period (4th-3rd century BC), there appears to be no strong correlation between monumental offerings and exercise of political power in the city. At the beginning of the 2nd century $\mathrm{BC}$, even as the democratic nature of the institutions is no longer contested, the top of the Delphian political notability decides to project itself through monuments celebrating the unity of the family and the excellence of its members. The concentration of political power in a narrow group during the second half of the 1st century BC could rely on practices of social distinctions already common for over a century. 
À PROPOS DE L'AUTEUR

N. KyriaKIdIs, université Paris-VIII.

\section{NOTE LIMINAIRE}

Une première version de ce travail a été présentée au colloque organisé par G. Biard et L. Thély, La construction de l'image publique dans l'Antiquité gréco-romaine (université Paris-Est, 28-29 mai 2010); j'ai eu l'occasion de revenir sur cette documentation lors du séminaire de M. Dana et I. Savalli, «Épigraphie et société dans le monde hellénistique » (ANHIMA, Paris, 27 octobre 2010). Je remercie $\mathrm{M}^{\text {mes }}$ A. Jacquemin et Chr. Müller ainsi que MM. G. Biard, R. Bouchon, D, Laroche, Fr. Lefevre, B. Holtzmann, D. Mulliez et D. Rousset pour leurs remarques. Toutes les imperfections de ce texte sont de ma seule responsabilité.

Je ne donne pas ici les références détaillées de l'exercice de fonctions civiques, sauf cas particulier. Le lecteur peut les retrouver aisément : pour les fonctions de prytane et de membre de la commission financière exceptionnelle, par une recherche sous le nom du titulaire dans l'index de CID II ; pour les fonctions de bouleute, d'archonte et de prêtre d'Apollon Pythien, grâce à l'index de G. DAux, Chronologie delphique (1943). C'est à la nomenclature des archontes éponymes établie par ce dernier ouvrage que renvoient les mentions B21, D3, K6, L30, N9 vel. sim.

Sauf mention contraire, les dates s'entendent avant J.-C.

ABRÉVIATIONS BIBLIOGRAPHIQUES

Les abréviations utilisées sont celles du Guide de l'Épigraphiste et de L'Année philologique.

DauX, Delphes = G. DAuX, Delphes au II' et au Ier siècle, depuis l'abaissement de l'Étolie jusqu'à la paix romaine, 191-31 av. J.-C., BEFAR 140 (1936).

JaCQuemin, Offrandes = A. JACQuemin, Offrandes monumentales à Delphes, BEFAR 304 (1999).

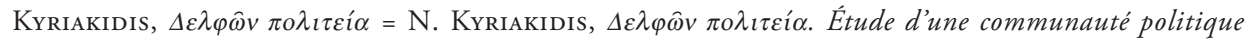

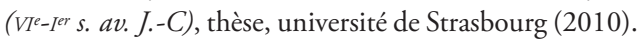

MA, Statues = J. MA, Statues and Cities. Honorific Portraits and Civic Identity in the Hellenistic World (2013). 
Une part notable de la documentation épigraphique d'époque classique et hellénistique sur les personnes privées appartient à une catégorie souvent délaissée par les historiens, celle des dédicaces. Je ne parle bien entendu pas ici des historiens des religions qui ont largement exploité ces inscriptions, principalement sous l'angle des divinités destinatrices ${ }^{1}$, mais plutôt de ceux des sociétés. Cette documentation n’a trop souvent servi qu’à préciser des stemmata, comme si l'essentiel de son intérêt figurait dans la gravure et que les bases sur lesquelles se trouvaient ces inscriptions n'avaient jamais servi de support qu'à des textes et non à des offrandes. À l'inverse, les spécialistes d'architecture et de sculpture ont eu tendance à privilégier l'étude des bases constituées de plusieurs blocs sur lesquels ils pouvaient restituer ou replacer des œuvres importantes pour l'histoire de l'art, négligeant trop souvent les vestiges les plus modestes et plus souvent encore le contexte social et politique qui avait présidé à leur production ${ }^{2}$. Différentes études récentes sont heureusement venues montrer tout l'intérêt qu'il y avait à inclure les offrandes monumentales - tout particulièrement la statuaire honorifique - dans la mise en histoire des communautés antiques ${ }^{3}$. Le présent article pour sa part, né d'une recherche sur la cité des Delphiens comme communauté politique du $\mathrm{VI}^{\mathrm{e}}$ au $\mathrm{e}^{\mathrm{er}} \mathrm{s}$., se veut une contribution à une histoire sociale de la pratique dédicatoire dans le monde des poleis classiques et hellénistiques ${ }^{4}$.

1. Sur les Delphiens et leurs dieux, voir JaCQuemin, Offrandes, p. 39-47 et A. JaCQuemin, « Panthéon et épiclèse delphiques : Apollon et les autres dieux ", dans N. Belayche, P. Brulé, G. Freyburger (éds), Nommer les Dieux. Théonymes, épithètes, épiclèses dans l'Antiquité (2005), p. 241-253.

2. Le volume très soigné de K. KIssas, Die attischen Statuen- und Stelenbasen archaischer Zeit (2000), est principalement consacré à l'architecture des bases et étudie secondairement la statuaire supportée, à l'exclusion de tout commentaire sur les dédicants (voir par exemple B45, p. 116-117 ou B70, p. 136).

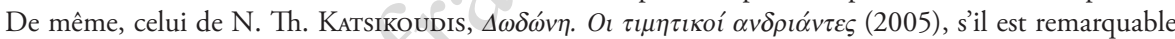
par sa volonté de traiter l'offrande comme un tout, ne s'aventure guère au-delà de l'étude strictement archéologique sauf pour des considérations générales sur l'histoire du sanctuaire.

3. Voir par exemple les études de R. KRUMeich, Bildnisse griechischer Herrscher und Staatsmänner im 5. Jahrhundert v. Chr (1997) et surtout "Formen der statuarischen Repräsentation römischer Honoranden auf der Akropolis von Athen im späten Hellenismus und in der frühen Kaiserzeit ", dans

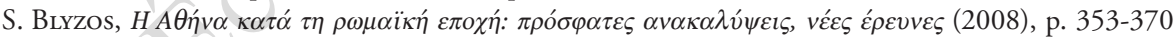
et R. Krumeich, Chr. Witschel, "Hellenistiche Statuen in ihrem räumlichen Kontext: Das Beispiel der Akropolis und der Agora von Athen", dans A. Matthaei, M. Zimmerman (éds), Stadtbilder im Hellenismus, Die hellenistische Polis als Lebensform 1 (2009), p. 173-226. Je n'ai pris connaissance de l'ouvrage de MA, Statues, qu'au moment où j'achevais ces lignes. Il est à espérer que le canevas global qu'il propose sur les statues honorifiques suscitera de nombreuses études de cas qui viendront préciser comment chacune des cités du monde grec a connu, ou non, les phénomènes qu'il décrit. Ma contribution pourra aussi être lue en ce sens, puisqu'elle traite également de cette documentation.

4. Je prolonge ici les conclusions de ma thèse : chap. III « Les acteurs de la vie civique », dans Kyriakidis, $\Delta \varepsilon \lambda \varphi \hat{\omega} v \pi 0 \lambda \imath \tau \varepsilon i ́ \alpha$, p. $240-440$, où j'ai tenté une sociologie de l'exercice du pouvoir politique à Delphes - compris comme la capacité et le droit de parler et d'agir au nom de la communauté - afin de déterminer dans quelle mesure le régime politique local était fermé ou ouvert. Une enquête dans ce domaine prend donc la forme d'une prosopographie des principaux acteurs de la vie civique (magistrats, bouleutes, intervenants à l'Assemblée, ambassadeurs) et d'une étude du recrutement à ces différentes fonctions, 
Pour préciser le contexte de cette documentation, peut-être ne sera-t-il pas inutile de rappeler quelques caractéristiques de la cité-État des Delphiens, qui est bien moins connue que l'illustre sanctuaire sis sur son territoires. Delphes était une petite cité; sa chôra, qui allait de la haute vallée du Pleistos à la plaine d'Amphissa, pouvait se traverser en quelques heures de marche ${ }^{6}$. Le nombre de citoyens - qui a dû varier dans le temps - n’est pas précisément connu, mais il s'établissait, dans une estimation large, entre 500 et 1500 mâles adultes - un chiffre comparable, quoique moindre, à celui de la population de Délos au $\mathrm{III}^{\mathrm{e}}$ s. ${ }^{7}$. La présence sur son territoire d'un sanctuaire "panhellénique " nous permet de disposer de sources exceptionnellement abondantes pour une si petite communauté, grâce notamment à trois séries documentaires : les comptes de construction du temple d'Apollon, au IV ${ }^{\mathrm{e}}{ }^{8}{ }^{8}$; les décrets de proxénie, du IV $\mathrm{Iu}^{\mathrm{e}} \mathrm{I}^{\mathrm{er}}$ s. et au-delà ${ }^{9}$; les actes d'affranchissement, enfin, à partir des dernières années du $\mathrm{III}^{\mathrm{e}} \mathrm{s}$. et jusqu’à l'époque impériale ${ }^{10}$.

En l'absence de presque toute source narrative et face à une documentation limitée pour l'essentiel à des noms de magistrats ou de parties représentées dans les actes d'affranchissement - hormis quelques unica épigraphiques comme les fondations attalides, il convient de se tourner vers les offrandes monumentales des Delphiens pour essayer de donner une profondeur socio-économique à la vie politique de la cité. Au vu de la difficulté d'établir des critères pour désigner tel ou tel citoyen qui apparaît dans nos sources comme faisant partie d'une classe sociale privilégiée ${ }^{11}$, son appartenance au groupe des dédicants d'offrandes monumentales constitue un moyen d'approcher le rapport entre excellence sociale (et économique) et exercice du pouvoir politique. Par l'expression "offrande monumentale » que j'emprunte à A. Jacquemin, j'entends ici les

mais aussi des réseaux - famille, groupe politique - par lesquels ces citoyens s'inséraient dans la communauté et parvenaient à y tenir une place privilégiée, au-delà du droit commun à participer à l'Assemblée.

5. Dans l'attente de la publication de l'étude citée à la note précédente, la meilleure présentation se trouve chez J. Oulhen, "Delphoi ", dans M. H. Hansen, T. H. Nielsen (éds), An Inventory of Archaic and Classical Poleis (2004), p. 412-416.

6. Sur le territoire de Delphes, voir D. RoussET, Le territoire de Delphes et la terre d'Apollon, BEFAR 310 (2002).

7. Ces chiffres sont tirés pour l'essentiel de quelques mentions du nombre des suffrages à l'Assemblée du peuple au IV e s. et d'estimations du nombre de participants aux fêtes des fondations attalides, au $\mathrm{II}^{\mathrm{e}} \mathrm{s}$. Voir Ph. Gauthier, "Les institutions politiques de Delphes au II siècle a. C. ", dans A. JacQuemin (éd.), Delphes cent ans après la grande fouille: essai de bilan. Actes du colloque international organisé par l'École française d'Athènes, Athènes-Delphes, 17-20 septembre 1992, BCH Suppl. 36 (2000), p. 112-113;

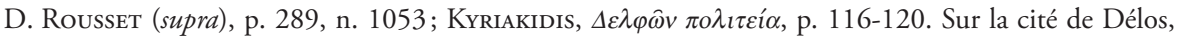
voir Cl. VIAL, Délos indépendante (314-167 av. J.-C.) : étude d'une communauté civique et de ses institutions, BCH Suppl. 10 (1984), p. 17-20.

8. J. Bousquet, Les comptes du quatrième et du troisième siècle, CID II (1989).

9. R. Bouchon, M. Douthe et moi-même avons la charge d'en publier le corpus (CID VI).

10. Je remercie D. Mulliez d'avoir bien voulu me permettre de consulter le manuscrit de son corpus sur Les actes d'affranchissement, CID V (à paraître).

11. Sur les quelques indices disponibles à Delphes, voir KYriakidis, $\Delta \varepsilon \lambda \varphi \hat{\omega} v \pi \circ \lambda \iota \tau \varepsilon i ́ \alpha$, p. 400-439. 
objets précieux offerts à la divinité - notamment les statues, mais aussi les reliefs votifs - assez importants pour avoir été installés, généralement en plein air, sur un support architectural portant une dédicace inscrite qui mentionne le nom du dédicant. Cette catégorie dépasse donc les typologies habituelles pour rapprocher des offrandes différentes (ex-voto, groupes familiaux, etc.) qui ont en commun d'être des dédicaces pérennes de monuments coûteux. Si ces monuments ne sont pas publics par leur dédicant (et donc leur financement), ils sont établis « en public » dans les espaces sacrés de la cité des Delphiens et prennent à témoin le passant de la piété de leur ordonnateur. Précisons que tous ces vestiges ont malheureusement été trouvés hors contexte, généralement en remploi dans le village moderne de Kastri, de sorte qu'il n'est pas possible d'étudier leur agencement dans l'espace delphique. Si la plupart d'entre eux devaient être implantés dans le hiéron d'Apollon Pythien, certains pouvaient avoir été érigés dans d'autres sanctuaires, tant à l'intérieur (Asklépiéion) qu'à l'extérieur du téménos (héroôn de Phylakos) ${ }^{12}$.

L'objet de cet article est donc d'examiner la manière dont les milieux favorisés d'une petite cité, Delphes, se sont mis en scène dans leur rapport au divin, faisant ainsi entrer un acte de piété dans une stratégie de distinction sociale pouvant contribuer à assurer leur prééminence dans cette communauté.

La période étudiée ici se situe entre le milieu du Ive s., époque à laquelle apparaissent les premiers monuments de Delphiens en même temps que la documentation sur la vie civique se fait plus abondante, et la fin du ier $\mathrm{s}$. qui correspond, nous le verrons, à une rupture dans le fonctionnement politique de la cité et à une évolution significative dans la manière dont l'excellence sociale est mise en scène.

Après avoir montré en quoi le groupe des dédicants de ces offrandes constitue une élite socio-économique et présenté un échantillon représentatif des éléments les mieux conservés de ce corpus, nous verrons que la confrontation de cette documentation avec les données dont nous disposons sur le régime politique delphique permet de dégager des évolutions qui ressortissent tout autant à l'histoire des pratiques religieuses et artistiques qu’à celles de la vie sociale et politique de la communauté étudiée.

\section{LE GROUPE DES DÉDICANTS COMME ÉLITE SOCIALE}

L'offrande monumentale n'est pas seulement un acte de piété pérenne, mais aussi une marque d'ostentation sociale. Le dédicant, en choisissant de dépenser ainsi une partie de ses ressources, ne se contente pas d'entrer en relation avec le divin : il le fait au vu

12. Sur la topographie des sanctuaires de Delphes, voir JACQUEMIN, Offrandes, particulièrement les p. 251-252. 
et au su de sa communauté, montrant ainsi sa capacité à réaliser des dépenses importantes non pas dans une finalité productive (chrématistique), mais dans le but le plus honorable qui soit, rendre hommage aux dieux. Contrairement à un sacrifice ou au cadeau d'un objet précieux qui doit être mis sous abri et donc soustrait au regard du plus grand nombre, l'offrande monumentale s'ancre dans l'espace du sanctuaire et défie l'oubli. Ainsi, l'exceptionnel sacrifice de cent vingt bœufs par Hermésandros à Cyrène ne nous est connu que par le soin qu'a pris son commanditaire de le commémorer par un sèma inscrit ${ }^{13}$. Seules ces offrandes ont laissé des traces susceptibles d'étude, puisque les inventaires de Delphes ne sont pas parvenus jusqu'à nous. Ce n'est cependant pas uniquement par nécessité que l'enquête se porte exclusivement sur celles-ci : ce type d'offrandes constitue une catégorie cohérente qui importe spécifiquement à mon propos et justifie la démarche adoptée, puisque les matériaux utilisés (la pierre et le bronze) et la présence d'une inscription concourent à la pérennité de l'acte d'offrir.

Leur coût, notamment lorsqu'elles comprennent de la statuaire, peut être assez élevé. On estime ainsi qu'à l'époque hellénistique un monument gravé supportant une statue de bronze à échelle humaine peut coûter entre 2000 et 3000 drachmes approximativement, soit, $\mathrm{au} \mathrm{II}{ }^{\mathrm{e}}$ s., le prix de sept à quatorze esclaves ordinaires à Delphes ou trois à quatre ans de salaire pour l'architecte de Délos ${ }^{14}$. À titre de comparaison, à l'Asklépieion d'Athènes, le poids en argent des offrandes varie d'une obole à 500 drachmes. Les offrandes pesant plus de 100 à 150 drachmes sont cependant presque exclusivement le fait du peuple athénien ou de magistrats dans l'exercice de leurs fonctions ${ }^{15}$. Le groupe de ces dédicants, au moins en ce qui concerne les offrandes les plus importantes, définit ainsi une catégorie d'individus appartenant à une élite économique qui se pense, se comporte et se met en scène comme une élite sociale, rivalisant ainsi de ses (modestes) moyens avec les Grecs fortunés venus de tout le pourtour méditerranéen pour laisser un témoignage de leur piété à Delphes. La recherche de distinction est particulièrement forte lorsque

13. Pour le texte grec, une traduction anglaise et la bibliographie, voir F. T. VAn Straten, "Gifts for the Gods ", dans H. S. Versnel (éd.), Faith, Hope and Worship. Aspects of Religious Mentality in the Ancient World, Studies in Greek and Roman Religion, vol. 2 (1981), p. 69 et n. 17 (p. 68).

14. Voir É. Perrin-Saminadayar, "Aere perennius. Remarques sur les commandes publiques de portraits en l'honneur des grands hommes à Athènes à l'époque hellénistique : modalités, statut, réception ", dans Y. Perrin, Th. Petit (éds), Iconographie impériale, iconographie royale, iconographie des élites dans le monde gréco-romain (2004), p. 114, pour plusieurs exemples et la n. 22 pour la bibliographie antérieure, et, dernièrement, MA, Statues, p. 245 et 264-265.

15. Voir S. B. Aleshire, The Athenian Asklepieion. The People, their Dedications, and the Inventories (1989), p. 47-48 et Asklepios at Athens. Epigraphic and Prosopographic Essays on the Athenian Healing Cults (1991), p. 75. Ces éléments de prix doivent donc amener à nuancer la remarque qui pouvait paraître de bon sens de ce même auteur dans The Athenian Asklepieion, p. 54, qui note qu'on ne peut considérer par principe qu'une offrande en pierre est plus coûteuse qu'une offrande en argent. 
l'objet de la dédicace est une statue du dédicant ou de ses proches ${ }^{16}$, pratique d'autant plus frappante qu’à Delphes, la cité n'honore pas ses citoyens de telles récompenses avant l'époque impériale. Ces monuments peuvent alors porter la revendication implicite d'être mis sur le même plan que les plus importants des bienfaiteurs étrangers, ceux à qui la communauté vote l'érection d'une statue ${ }^{17}$.

\section{QUELQUES EXEMPLES D'OFFRANDES DE DELPHIENS}

Il n'est pas question ici de proposer un corpus de ces offrandes, d'autant plus que ces documents sont généralement bien publiés ${ }^{18}$. La présentation de quelques exemples permettra de montrer que ce matériel, bien que limité quantitativement, pauvre comparé à celui des riches cités d'Asie Mineure, trouvé hors contexte et systématiquement privé des offrandes statuaires dont il était le support, a néanmoins quelque chose à nous apprendre sur la société des Delphiens.

\section{Du IVe au III' $s$.}

- Au milieu du rve s., Damaios fils d'Antidôros offre une statuette en bronze installée sur une base circulaire en calcaire $($ h. $0,59 \mathrm{~m}$; d. $0,365 \mathrm{~m}$; mortaises : long.

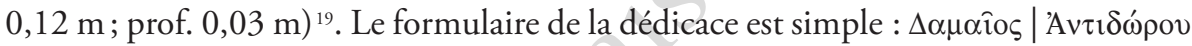
| $\alpha \dot{v} \varepsilon \dot{\theta} \eta \kappa \varepsilon$, «Damaios fils d'Antidôros a consacré (cette offrande) $»^{20}$. Ni Damaios ni Antidôros n'ont été archontes. Damaios est vraisemblablement - le nom est rare - le père de deux Delphiens actifs politiquement. L'un, Achaiménès, est membre d'une commission financière exceptionnelle en 326/5 sous l'archonte Charixénos (C27) et peut avoir aussi été bouleute et archonte; l'autre, Aristônymos, est prytane en 319/8 sous Éribas (D2), il peut avoir déjà été soit prytane soit archonte (C17) en 341/0 et peut-être aussi membre de la même commission sous Charixénos. L'offrande n'est pas des plus riches si on la compare à celle d'Asôpodôros étudiée plus bas, mais elle

16. Voir MA, Statues, notamment p. 225-233.

17. Sur les bénéficiaires des statues votées par les Delphiens : JACQUEmin, Offrandes, p. 39-44.

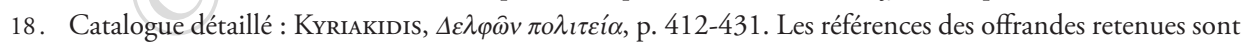
les suivantes : JACQUEmin, Offrandes, nos 229, 230, 232, 221, 231, 224, 225, 226, 235, 217, 228, 233, 220, 227, 237, 253, 219, 250bis, 269, 264, 261, 242, 218, 262, 234, 239, 014, 249, 223, 241, 236 (mais sans doute pas le no 263, comme D. Rousset a bien voulu me le faire remarquer : voir Addenda à ma thèse); FD IV 6, n ${ }^{\text {os }}$ 5-8; FD III 1, 487-495. Pour une présentation générale des offrandes privées des Delphiens, voir JACQUEMIN, Offrandes, p. 44-46.

19. Inv. 1758. H. Ромтоw, "Delphische Neufunde II ", Klio 15 (1918), p. 65, no 91 ; KyriaKidis, $\Delta \varepsilon \lambda \varphi \omega \hat{\omega}$

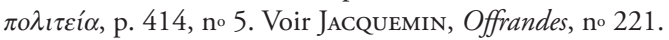

20. Sur ces questions: A. Jacquemin, "Ordre des termes des dédicaces delphiques », AION 2 (1995), p. 141-157. 
témoigne d'une certaine aisance chez un personnage vraisemblablement apparenté à des citoyens ayant exercé des responsabilités politiques.

- Au milieu ou lors de la seconde moitié du IV s., Tarantinos et Pitthis dédient à Dionysos une offrande indéterminée supportée par un pilier de calcaire bleuté à moulures (h. cons. 0,21 m; 1. 0,40-0,297 m; ép. 0, 39-0,29 m; trou d'encastrement

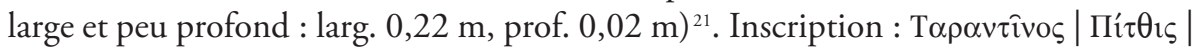

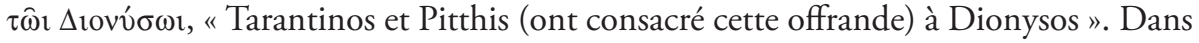
les comptes, deux prytanes portent le nom de Tarantinos (sous les archontes C15 et C17 : 343/2 et 341/0) ainsi qu'un bouleute sous les archontes C19, C29 (327 à 323)

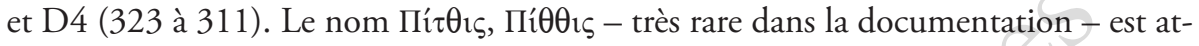
testé dans les collèges de bouleutes B4 et B10 (deuxième quart du IV ${ }^{\mathrm{s}} \mathrm{s}$.). On aimerait connaître les liens qui unissaient les deux dédicants (parenté? amitié?). Aucun de ces deux personnages n’a été archonte et s'ils exercent plusieurs responsabilités politiques, ils ne sont pas les plus présents de leur génération dans la documentation, de même que leur offrande ne se distingue pas par sa richesse.

- À la fin du IV s. ou au début du $\mathrm{III}^{\mathrm{e}}$ s., Hagestratos dédie à Apollon une offrande installée sur une base de marbre à moulures (h. 0,08 $\mathrm{m}$; 1. 0,275-0,262 $\mathrm{m}$; ép. cons.

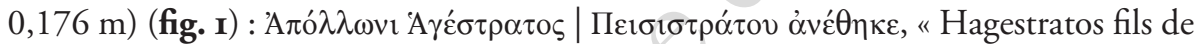
Peisistratos a consacré (cette offrande) à Apollon "22. La base comporte une cuvette ovale $(0,21 \mathrm{~m}$ sur peut-être $0,12 \mathrm{~m})$ pour statuette ou offrande en pierre. Le nom d'Hagestratos n'est attesté pour aucun archonte ou bouleute connu. Aucun Peisistratos

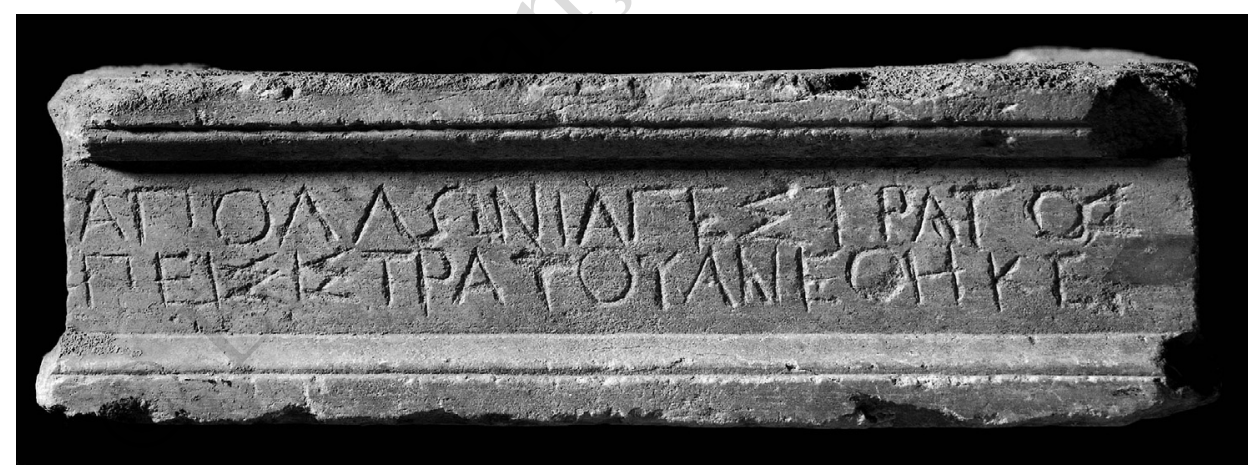

Fig. I — Base d'Hagestratos fils de Peisistratos (cl. EFA, Ph. Collet).

21. Inv. 3321. Th. Homolle, "Inscriptions de Delphes (II)", BCH 23 (1899), p. 530; G. Daux, J. Bousquet, "Agamemnon, Télèphe, Dionysos Sphaléotas et les Attalides II ", RA 20 (1942/3),

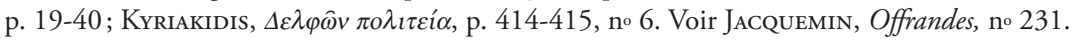

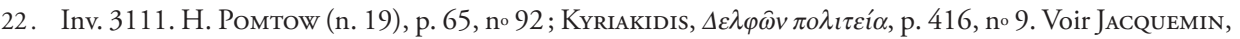
Offrandes, no 224. 
non plus n'est mentionné à Delphes dans les comptes du IV s. Un Lysidamos fils d'Hagestratos est bouleute en D4 (de 323 à 311). S'il n'est pas impossible que ces Delphiens aient exercé quelques fonctions dans les lacunes de la documentation, ni le dédicant de cette modeste offrande ni son père n'ont assurément exercé l'archontat.

- Vers la même époque, une dénommée [...]eboula commémore sa sortie de charge de la prêtrise d'Ilithyie par l'offrande d'une statuette en pierre sur un cippe de brèche rouge à moulures $(\mathrm{h} .0,44 \mathrm{~m} ; 1.0,24-0,25 \mathrm{~m}$; ép. $0,21 \mathrm{~m}$; petite cuvette ovale : $0,145 \mathrm{~m}$ sur $0,09 \mathrm{~m}):[\ldots$.

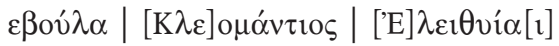

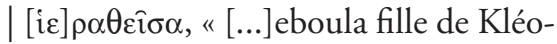
mantis (a consacré cette offrande) à Ilithyie après avoir exercé sa prêtrise $»^{23}$. On ne connaît d'offrandes de sortie de charge que pour cette divinité, encore ne s'agit-il que de trois cas, du IV $\mathrm{s}$. jusqu'au milieu du $\mathrm{II}^{\mathrm{e}} \mathrm{s}$. La dédicante est peut-être la fille d'un prytane sous l'archontat D3 (324 à 314) dénommé Kléomantis (et non Kleumantis comme indiqué de manière erronée dans l'édition de référence $)^{24}$. Ce nom est attesté dans plusieurs collèges de bouleutes à la fin du IV ${ }^{\mathrm{e}}$ s. et au début du III ${ }^{\mathrm{e}}$ s. (F6, après 315; F25 ca 290-280).

- À la fin du Ive s., une grande base de statue en calcaire (h. $0,42 \mathrm{~m}$; l. cons. $0,34 \mathrm{~m}$; ép. $0,65 \mathrm{~m})^{25}$ est signée par le bronzier Daitôndas de Sicyone (fig. 2). La dédicace est la suivante:

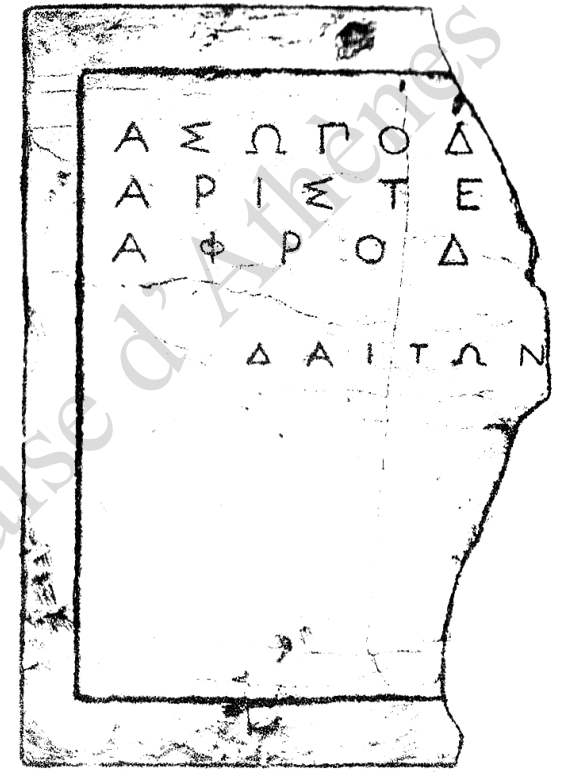

Fig. 2 - Base signée par Daitôndas de Sicyone. D’après H. Poмтоw, Beiträge zur Topographie von Delphi (1889), pl. XIV 47.

23. Inv. 1114. J. Bousquet, «Inscriptions de Delphes (XI) », BCH 87 (1963), p. 190 et fig. 2; Kyriakidis, $\Delta \varepsilon \lambda \varphi \hat{\omega} v \pi 0 \lambda \imath \tau \varepsilon i ́ \alpha$, p. 417, no 12. Voir JaCQuemin, Offrandes, no 235. Pour la trad. voir n. 47.

24. Une vérification sur la pierre m'a permis de constater que ce nom apparaissait bien sous la forme commune Kléomantis et non sous la forme dialectale Kleumantis (lapsus de J. BousQuet [n. 23], p. 190).

25. Sans numéro d'inventaire. J. Schмidt, «Reisefrüchte (Schluss.) ", MDAI (A) 5 (1880), p. 197-198, no 58; H. Ромтоw, Beiträge zur Topographie von Delphi (1889), p. 83-84 et pl. XIV 47 (H. Ромтоw, "Delphica III. Vorläufiger Bericht über die Ergebnisse einer Reise nach Delphi [2] ", BPhW [1912], col. 93/p. 54; H. Ромтоw, «Die Kultstätten der 'anderen Götter' von Delphi », Philologus 71 [1912], p. 37-38) ; J. MARCADÉ, Recueil des signatures de sculpteurs grecs, vol. 1 (1953), 25 (FD III 4, p. 148, n. 4); Kyriakidis, $\Delta \varepsilon \lambda \varphi \hat{\omega} v \pi$ o $\lambda \tau \tau \varepsilon i ́ \alpha$, p. 417-418, no 13. Voir JaCQuemin, Offrandes, no 217. 


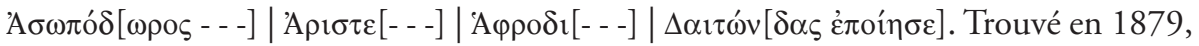
ce bloc a malheureusement été perdu avant 1953. Je n’entre pas ici dans le débat sur les différentes restitutions de la dédicace qui ont été proposées : aucune ne s’impose de manière indiscutable ${ }^{26}$. Notons simplement qu'il existe plusieurs solutions : Asôpodôros est le fils d'Aristeidas et a fait à Aphrodite la consécration d'une statue représentant la déesse ou une figure liée à son culte; Asôpodôros et Aristeidas sont tous les deux dédicants et leur patronyme se situait dans la lacune; ou encore l'un des deux est le dédicant (au nominatif) d'une statue de l'autre (à l'accusatif), statue consacrée à Aphrodite ${ }^{27}$.

Il s'agit de la seule occurrence du travail à Delphes de ce sculpteur connu par ailleurs ${ }^{28}$. Cette offrande se distingue des précédentes et peut passer pour particulièrement riche dans le contexte delphien de son temps : ses dimensions permettent d'y restituer au moins une grande statue en bronze. Les deux noms d'Aristeidas et d'Asôpodôros reviennent dans les inscriptions de Delphes de la fin du $\mathrm{IV}^{\mathrm{e}}$ s. et du début du $\mathrm{III}^{\mathrm{e}}$ s., notamment pour deux bouleutes de l'archontat de Lyson (F11). Un Asôpodôros est prytane en $331 / 0$ sous Thyméas et en 327/6 sous Caphis (C26). Ce nom est porté aussi par des bouleutes à la même période (quatre semestres : C24 [329/8], E3 [321/0], D7 [314/3], F11 [après 315]). Un Asôpodoros fils de Sakedallos est naope dans les dernières années du IV e s. ${ }^{29}$. Un Aristeidas est prytane sous D3 (324 à 313, 318/7 ?) et un bouleute porte ce nom sous D6 (321 à 307, 315/4?) et F11 (après 315). Aucun des deux personnages n'a été archonte. Néanmoins, la possible identification du premier personnage de cette inscription avec Asôpodoros fils de Sakedallos est assez intéressante, car cette fonction amphictionique non limitée dans le temps a été exercée par des gens en vue dans leurs cités $^{30}$. Nous aurions alors la rencontre d'une carrière politique bien remplie et d'une offrande relativement plus riche que les autres.

$\mathrm{Au} \mathrm{III}^{\mathrm{e}}$ s., les offrandes sont plus rares et présentent peu de différences, à l'exception d'une attestation isolée et mal datée de la dédicace d'une statue représentant une prêtresse à l'occasion de sa sortie de charge qui est le premier cas connu de figuration d'un mortel

26. L'édition de référence est celle J. MarCadÉ (supra), où figurent les différentes propositions de restitution.

27. Cette dernière hypothèse est moins probable. Je ne retiens pas la solution proposée par J. Pouilloux dans FD III 4, p. 148, n. 4, qui veut faire d'A elle se heurte à l'absence dans l'onomastique delphique de noms composés sur celui de la déesse Aphrodite avant l'époque impériale.

28. On sait par Pausanias, 6, 17, 5, qu'il était de Sicyone et qu'il avait fait à Olympie la statue d'un jeune boxeur, Théotimos d'Élis fils de Moschiôn. Il est également connu par une base signée de Thèbes (IG VII, 2472) qui comporte des traces d'arrachement d'une statue en bronze.

29. CID II 119, 1. 38. Sur ce personnage, voir KYrIAKIDIs, $\Delta \varepsilon \lambda \varphi \hat{\omega} v \pi o \lambda \iota \tau \varepsilon i ́ \alpha$, p. 285.

30. Fr. Lefèvre, L'Amphictionie pyléo-delphique. Histoire et institutions, BEFAR 298 (1998), p. $265-266$. 
delphien $^{31}$. Le début du $\mathrm{II}^{\mathrm{e}}$ s. voit une évolution significative, avec le retour d'une documentation plus abondante et l'apparition de monuments familiaux.

\section{Au II et au I I $^{\text {er }}$.}

Au moins quatre dédicaces peuvent être attribuées à la famille de Praxias et de Praxô, une des plus importantes dans la Delphes du début du $\mathrm{II}^{\mathrm{e}}$ s. ${ }^{32}$.

- Au tout début du siècle, une première offrande est faite par Praxô (h. cons. 0,49 m;

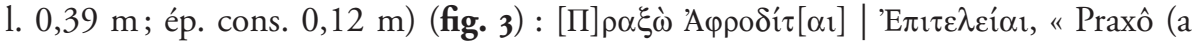
consacré cette offrande) à Aphrodite Épitéleia ${ }^{33}$. Il ne s'agit sans doute pas d'une stèle, comme on a pu l'écrire, mais plutôt d'une base de statue dont la face postérieure n'est pas conservée. La partie observable du lit d'attente est piquetée, mais ne présente aucune trace des mortaises qui devaient se trouver dans la partie perdue. Praxô était d'après Tite-Live l'hôtesse de Persée de Macédoine et appartenait à l'élite sociale et

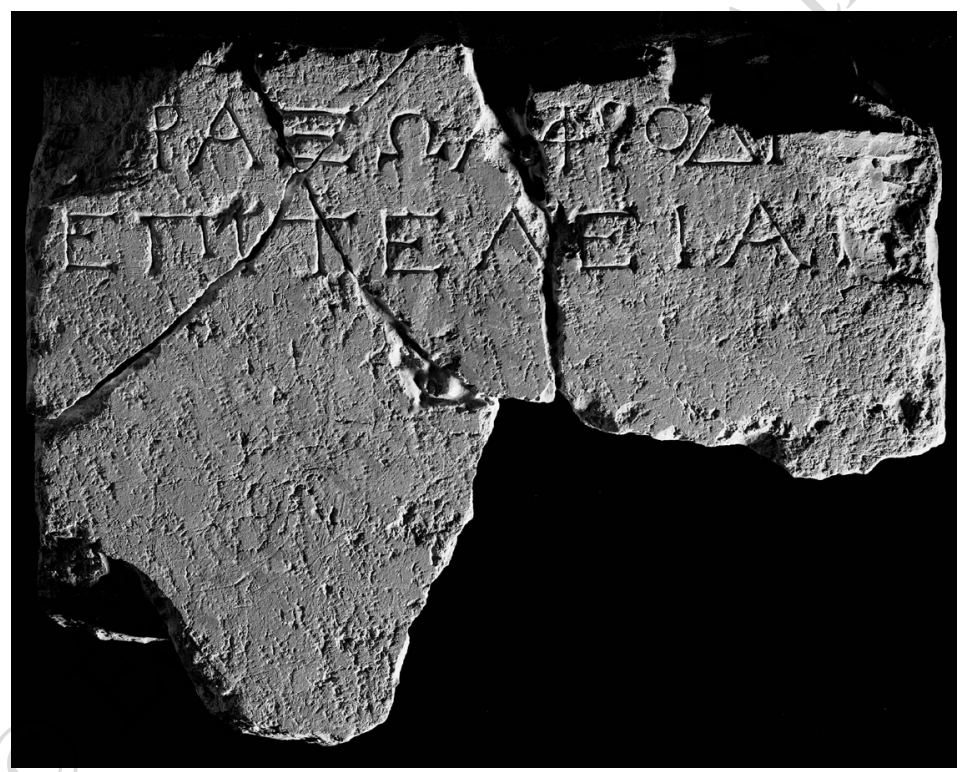

Fig. 3 - Offrande de Praxô à Aphrodite Épitéleia (cl. EFA, Ph. Collet).

31. Th. Homolle, "Inscriptions de Delphes (I) », BCH 23 (1899), p. 387-388; H. Ромтow dans Philologus 71 (n. 25), p. 41-42; J. BousQuet (n. 23), p. 189-190; Kyriakidis, $\Delta \varepsilon \lambda \varphi \hat{\omega} v \pi o \lambda \imath \tau \varepsilon i ́ \alpha$, p. 420, no 20. Voir JaCQuemin, Offrandes, p. 45 et n. $60 ;$ no 253.

32. Sur cette famille, voir DAUX, Delphes, p. 448-451 (avec stemma).

33. Inv. 1729+1773. P. De La Coste-Messelière, «Inscriptions de Delphes », BCH 49 (1925), p. 79, no 5 (J. Bousquet [n. 23], p. 188); J. Pouilloux, FD III 4, 468 et pl. 23D; Kyriakidis, $\Delta \varepsilon \lambda \varphi \hat{\omega} v \pi 0 \lambda \iota \tau \varepsilon i ́ \alpha$, p. 421, no 21; A. Jacquemin, D. Mulliez, G. Rougemont, Choix d'inscriptions de Delphes, traduites et commentées, ÉtÉpigr 5 (2012), no 159. Voir JaCQuemin, Offrandes, p. 45 et n. 64; no 219. 
économique de la cité ${ }^{34}$. On sait par ailleurs que son père s'appelait Emménidas (I), un nom porté par plusieurs Delphiens influents, dont des bouleutes de la seconde moitié du III ${ }^{\mathrm{e}}$ s. Son frère Kallias fils d'Emménidas (I) a été bouleute sous L9 (190/89) et ambassadeur auprès des Attalides avec son beau-frère Praxias fils d'Eudokos ${ }^{35}$. Praxô a épousé Praxias, qui est peut-être son parent.

- Praxias a offert à Apollon, vers 175, la statue de son fils Eudokos pour commémorer

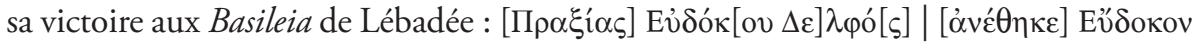

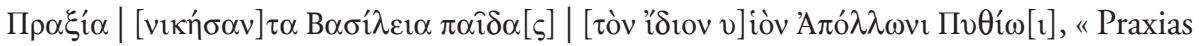
fils d'Eudokos de Delphes a consacré à Apollon Pythien (la statue) de son fils Eudokos fils de Praxias pour sa victoire aux Basileia dans le concours des paides $"{ }^{36}$. Les circonstances et le motif de la dédicace sont intéressants : il s'agit de la première attestation de l'offrande d'une statue d'athlète delphien vainqueur; elle est du père de l'athlète, qui choisit de donner une publicité et un éclat inaccoutumés à une victoire acquise dans un concours régional. Le dédicant est déjà un des personnages les plus en vue de sa cité, même s'il n'est pas encore au sommet de sa carrière. Après avoir été bouleute en 192/1 (L7), dans les derniers temps de la domination étolienne, il poursuit son activité sous l'hégémonie romaine : bouleute à nouveau en 188/7 (L11), ambassadeur auprès des Rhodiens dont il est le proxène en $180 / 79$ ou $179 / 8^{37}$, archonte en 178/7 (L21), à nouveau bouleute en 177/6 (L22) et 173/2 (L26), deux fois ambassadeur auprès des Attalides $c a 160^{38}$, désigné en 160/159 pour cinq ans comme épimélète de la fondation d'Attale pour l'instruction des enfants ${ }^{39}$, prêtre d'Apollon Pythien (collège VI : 153/2144/3). Son frère Thrasyklès, après avoir exercé le bouleutat au premier semestre de 190/89 (L9), fut ambassadeur à Rome en 189 (les ambassadeurs furent assassinés sur le chemin du retour ${ }^{40}$. Leur frère Sôsinikos doit très probablement être identifié avec le bouleute de 184/3 (L15) et 180/79 ou 179/8 (L20) et l'archonte de 174/3 (L25).

34. Tite-Live, 42, 15 (... litterasque eis dat ad Praxo hospitam, principem auctoritate et opibus Delphorum) et 17.

35. FD III 3, 239 (J. Poullloux, Choix d'inscriptions grecques : textes, traductions et notes : travaux de l'Institut d'épigraphie grecque (1960), no 12; H. KoTsıdu, Timè kai doxa: Ehrungen für hellenistische Herrscher im griechischen Mutterland und in Kleinasien unter besonderer Berücksichtigung der archäologischen Denkmäler (2000), no 95; A. Jacquemin, D. Mulliez, G. Rougemont [n. 33], no 166.

36. Inv. 30936. C. Wescher, P. Foucart, Inscriptions recueillies à Delphes (1863), no 477 (sans la 1. 1); H. Ромтоw, "Fasti Delphici (I)", Jahr. f. class. Phil. 57 (1889), p. 567, n. 73; Kyriakidis, $\Delta \varepsilon \lambda \varphi \hat{\omega} v$ $\pi \circ \lambda \imath \tau \varepsilon i ́ \alpha$, p. 421-422, no 22. Voir JaCQUemin, Offrandes, no 250bis [la base, provisoirement disparue, voir p. 330, n. 39, a été retrouvée].

37. FD III 3, 383; D. Rousset (n. 6), inscr. no 1.

38. Réf. à la n. 35 .

39. Daux, Delphes, p. 686 (J. Poullloux [n. 35], no 13; H. Kotsidu [n. 35], no 97; A. Jacquemin, D. Mulliez, G. Rougemont [n. 33], no 168).

40. FD III 4, 353 A et B. En dernier lieu, voir Chr. Haвicht, "Tod auf der Gesandschaftsreise ", dans B. Virgilio (éd.), Studi ellenistici XIII (2001), p. 14. 
- On connaît la base d'une statue de Thrasyklès fils de Praxias, offerte à Apollon Pythien à l'occasion d'une victoire (pythique?) au pancrace (h. 0,256 m; 1. 0,685 m; ép.

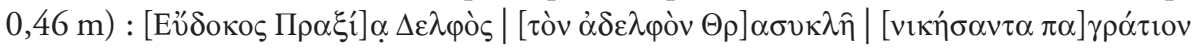

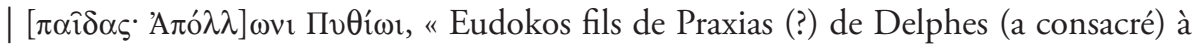
Apollon Pythien (la statue) de son frère (?) Thrasyklès pour sa victoire au pancrace dans le concours des paides "41. Sur l'unique bloc parvenu jusqu'à nous, on observe une mortaise pour semelle de pied gauche d'une statue de bronze à échelle humaine (long. 0,22 m, larg. de 0,04 à 0,06 m, prof. 0,05 m). P. de La Coste-Messelière a restitué, sans certitude, le nom de son frère Eudokos comme dédicant. Après avoir été honoré par son père pour une victoire, ce dernier aurait honoré son jeune frère d'une statue pour le même motif. Si Eudokos a été bouleute (L51 et L54, entre 151/0 et $147 / 6^{42}$ ), il ne semble jamais avoir été archonte. La personne honorée, Thrasyklès fils de Praxias, est archonte en L53 (entre 151/0 et 147/6) et bouleute en L50 (entre $153 / 2$ et 144/3). On ne doit donc pas imaginer une grande différence d'âge entre les deux frères si l'on accepte la restitution; ils parviennent l'un et l'autre à maturité politique alors que leur père est prêtre d'Apollon. Il faut noter qu'Eudokos aurait dédié la statue de son frère du vivant de leur père, perpétuant ce qui est dans cette documentation particulière une spécificité familiale, la célébration des victoires agonistiques des membres de la famille par l'offrande de leur portrait.

- Une dernière dédicace de cette famille est faite au troisième quart du II $\mathrm{s}$. par Praxô qui offre en ex-voto la statue de son petit-fils Praxias, fils du Thrasyklès que l'on vient

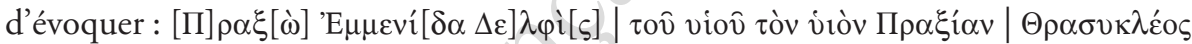

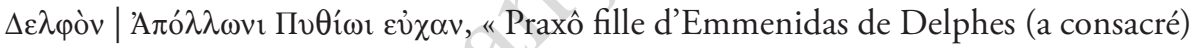
à Apollon Pythien (la statue) du fils de son fils Praxias fils de Thrasyklès de Delphes, en accomplissement d'un vœu " ${ }^{43}$. Le bloc isolé qui en témoigne et sur lequel tient toute la dédicace (h. $0,256 \mathrm{~m} ; 1.0,685 \mathrm{~m}$; ép. 0,46 m) appartenait à un ensemble plus grand (les faces latérales et postérieure sont travaillées pour joint; au lit d'attente, mortaises pour crampon et goujon), peut-être, comme cela a été supposé, un monument familial à plusieurs statues. Fils d'archonte, petit-fils d'archonte et de prêtre, Praxias fils de Thrasyklès n'a exercé aucune fonction politique connue. Il faut peut-être penser dans son cas à une mort précoce ou à une autre forme de retrait de la vie civique : présent aux côtés de ses frères Damainetos et Eudokos dans un acte d'affranchissement

41. Inv. 5558. P. de La Coste-Messelière (n. 33), p. 7, no 3. Kyriakidis, $\Delta \varepsilon \lambda \varphi \hat{\omega} v \pi o \lambda \imath \tau \varepsilon i ́ \alpha$, p. 421, no 23. Voir JaCQUemin, Offrandes, no 269.

42. Sur la datation des archontats du II s., voir en dernier lieu D. MulLiez, «Les fils d'Ariane. Quelques questions de chronologie delphique ", CRAI (2006), p. 2233-2255.

43. Inv. 4812. P. de La Coste-Messelière, (n. 33), p. 78, no 4 ; FD III 4, 246, pl. XXXIV, n॰ 2 ; Kyriakidis, $\Delta \varepsilon \lambda \varphi \hat{\omega} v \pi \circ \lambda \imath \tau \varepsilon i ́ \alpha$, p. 422-423, no 24; A. Jacquemin, D. Mulliez, G. Rougemont (n. 33), no 160. Voir JACQUEMIN, Offrandes, no 264. 
daté de $134 / 3^{44}$, il ne se porte pas accusateur en leur compagnie lors du scandale de ca $117^{45}$.

À la même époque, une autre famille se distingue par le nombre et la nature de ses offrandes ${ }^{46}$.

- Au début du Ii e s., avant 189, Xénôn et ses fils Boulôn, Mnasôn et Peithagoras offrent la statue de leur femme et mère Pasichon, qui vient d'exercer la charge de prêtresse d'Ilithyie (fig. 4). La base monolithe (h. 0,32 m; 1. 0,75 m; ép. 0,735 m)

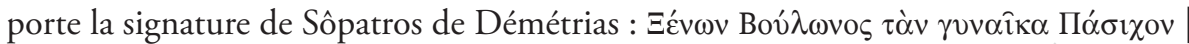

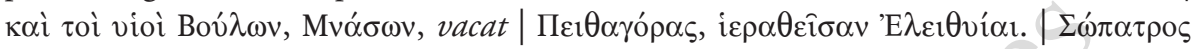

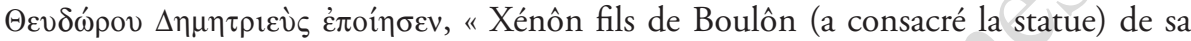
femme Pasichon à Ilithyie, après l'exercice de sa prêtrise, en compagnie de ses fils Boulôn, Mnasôn et Peithagoras. Sôpatros fils de Theudôros de Démétrias a fait (la statue) » ${ }^{47}$. Xénôn fils de Boulôn est prêtre d'au moins 200 jusqu’à sa mort en 180. En l'absence de mention du patronyme des archontes et des bouleutes au III ${ }^{e}$ s., la banalité de ce nom à Delphes interdit d'affirmer qu'il a exercé l'une de ces fonctions, même si les parallèles rendent ce fait hautement probable. Son fils Boulôn est l'un des ambassadeurs assassinés en 189 en compagnie du Thrasyklès, frère de Praxias, mentionné ci-dessus; il a probablement été bouleute en 195/4 (L4). Mnasôn, dont le nom est rare, doit sans doute être identifié avec le bouleute sans patronyme des archontats L5 (194/3), L9 (190/89) et peut-être encore L21 (178/7). Peithagoras pourrait être l'archonte de 194/3 (L5), mais il n'est pas connu comme bouleute. Il semble que les cinq membres de cette famille participent tous au fonctionnement de la cité. Le caractère nouveau de la démarche doit être souligné : il s’agit désormais de célébrer une sortie de charge en rendant hommage à la déesse, tout en offrant collectivement la statue d'un membre de la famille. Ce décentrement donne une nuance honorifique à l'exercice de la prêtrise : ce n'est plus le titulaire du sacerdoce qui remercie la divinité, mais ses proches qui célèbrent l'exercice de son sacerdoce. Le sculpteur est mentionné

44. SGDI 2194. Texte daté par l'archonte L63.

45. Cette question est traitée en détail dans Kyriakidis, $\Delta \varepsilon \lambda \varphi \hat{\omega} v \pi$ $\pi$ $\lambda \iota \tau \varepsilon i ́ \alpha$, p. 386-398. Les pièces du dossier ont été rééditées dans CID IV 118-119 où se trouvent les références à la bibliographie récente. Voir en dernier lieu D. Summa, «Ein Amphiktioniedekret aus Ostlokris. Neues zu "Le scandale de ca 117 av. J.-C." ", ZPE 172 (2010), p. 100-104.

46. Voir Daux, Delphes, p. $452-453$ (avec stemma).

47. Inv. 820. Th. Homolle (n. 31), p. 386 (Syll. ${ }^{3}$ 602); H. Ромтow, loc. cit. à la n. 25 (1912), p. 41;

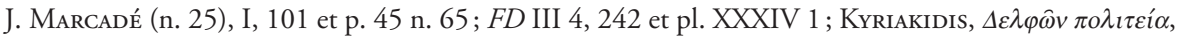
p. 423, no 25; A. Jacquemin, D. Mulliez, G. Rougemont (n. 33), no 142 [traduction légèrement différente de celle retenue ici; je considère que dans une dédicace le datif s'explique mieux comme complément du verbe sous-entendu indiquant l'offrande, d'autant plus que iepóouðı a généralement son complément au génitif, même si le datif est également, mais plus rarement, attesté]. Voir JaCQuemin, Offrandes, no 261. 


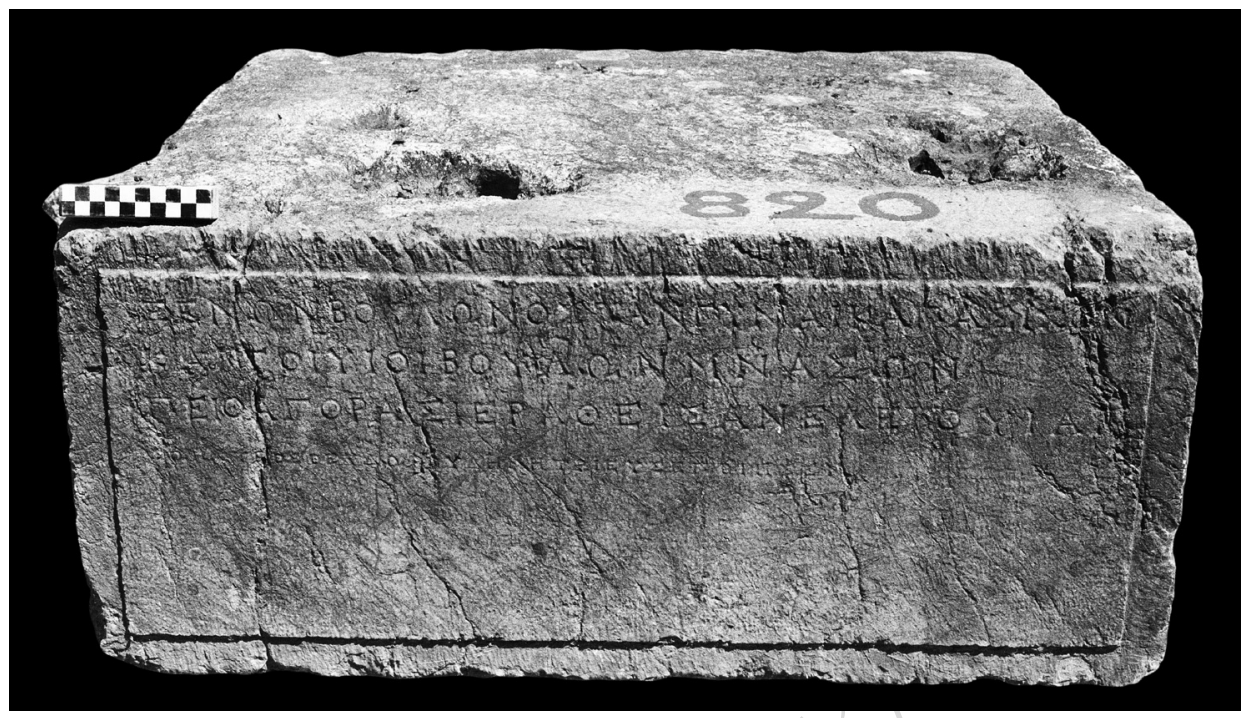

Fig. 4 - Statue de Pasichon offerte par son mari Xénôn et ses fils Boulôn, Mnasôn et Peithagoras (cl. EFA, D. Mulliez).

par deux autres inscriptions de Delphes. Il est notable que l'une d'elles soit la consécration à Apollon de la statue de leur père par deux Delphiens qu'il faut peut-être identifier à des bouleutes ${ }^{48}$.

- Au deuxième quart du II e s., Xénôn (II) fils de Boulôn (petit-fils du précédent) et sa femme Kleupatra fille de Chairéas offrent à Apollon Pythien la statue de leur fils Aristomachos (inv. 1763 : h. cons. 0,241 m; l. cons. 0,392 m; ép. cons. 0,274 m+

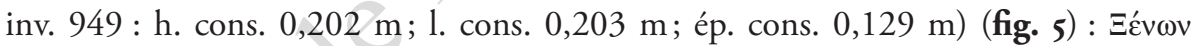

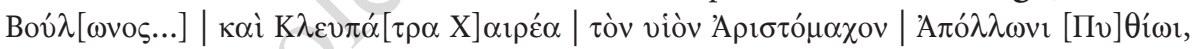
"Xénôn fils de Boulôn... et Kleupatra fille de Chairéas (ont consacré la statue) de leur fils Aristomachos à Apollon Pythien " ${ }^{49}$. Xénôn (II) a été bouleute en 169/8 (L30), 168/7 (L31) et 166/5 (L33); il est nommé, ca 160, épimélète pour cinq ans de la fondation d'Attale avec Praxias fils d'Eudokos et Bakchios fils d'Agrôn ${ }^{50}$. On peut affirmer qu’il n'a jamais été archonte. Son fils n'a jamais été ni archonte ni bouleute.

Cette famille qui a tenu un rôle politique de premier plan dans la cité lors de la première moitié du $\mathrm{II}^{\mathrm{e}} \mathrm{s}$. disparaît ensuite de la documentation.

48. H. Pomtow (n. 19), p. 53, no 74 (pour le fragment inv. no 641); FD III 1, 524; J. MarCadÉ (n. 25), I,

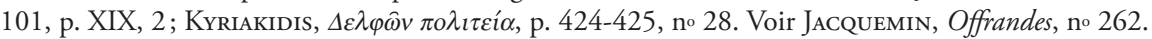

49. Daux, Delphes, p. 453; FD III 4, p. 282, n. 1 (corr. à l'ed. princ.); Kyriakidis, $\Delta \varepsilon \lambda \varphi \omega \hat{v} v \pi 0 \lambda \imath \tau \varepsilon i ́ \alpha$, p. 424, no 26. JACQUEMIN, Offrandes, no 242.

50. Références supra à la n. 39. 


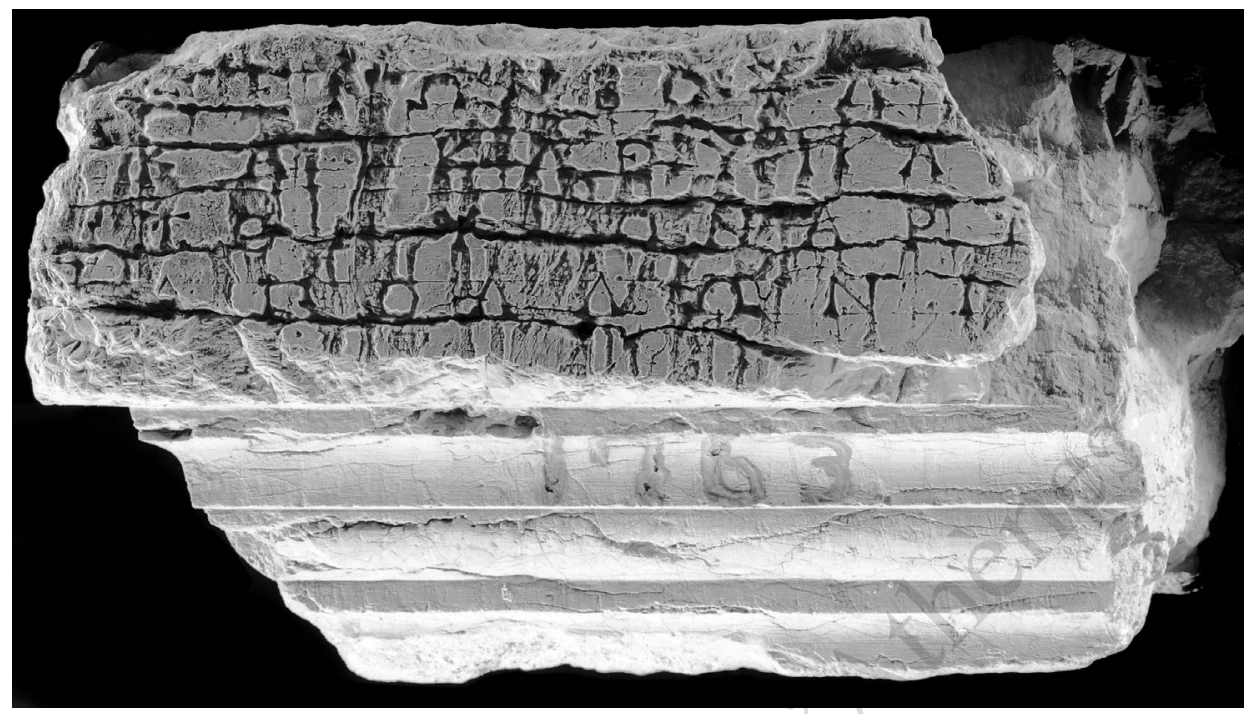

Fig. 5 - Statue d'Aristomachos offerte par ses parents, Xénôn (II) fils de Boulôn et Kleupatra fille de Chairéas (cl. EFA, Ph. Collet).

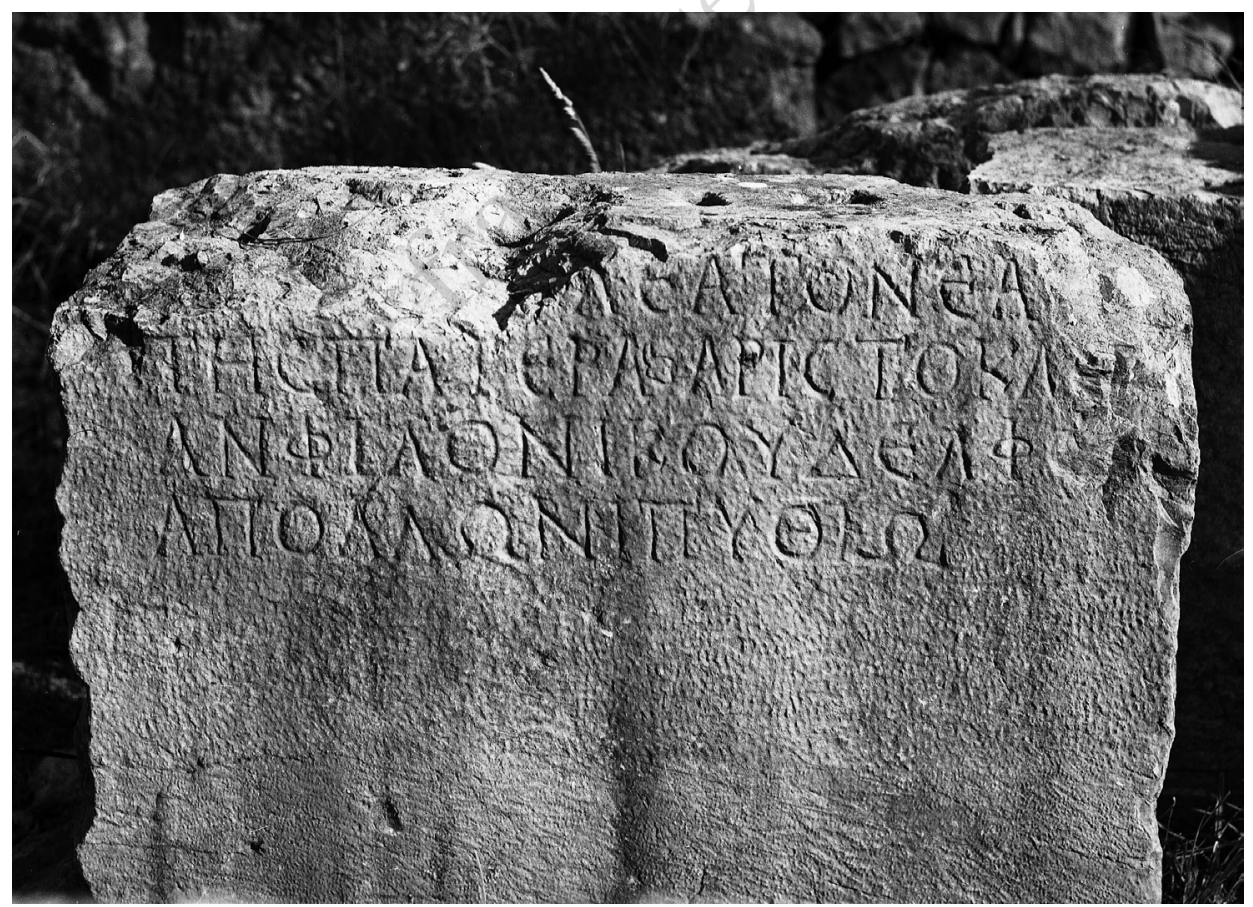

Fig. 6 - Statue d'Aristokléas fils de Philonikos offerte par sa fille [Aristok?]léa (cl. EFA, J. Bousquet). 
Au début du II $^{\mathrm{e}}$ s., dans un milieu sans doute très étroit, l'offrande de statues de membres de la famille est devenue une pratique courante. Les offrandes conservées sont plus rares pour la seconde moitié du $\mathrm{II}^{\mathrm{e}} \mathrm{s}$. et il n'y en a qu'une seule qui puisse dater de la première moitié du $\mathrm{I}^{\mathrm{er}} \mathrm{s}$. Il faut attendre les années 40-20 pour que la documentation soit à nouveau un peu plus abondante et l'on peut alors constater que la mode qui s'est imposée au début du $\mathrm{II}^{\mathrm{e}} \mathrm{s}$. se poursuit. Néanmoins, ces pratiques se perpétuent dans une cité dont les cadres institutionnels et, sans doute, socio-économiques ont été bouleversés.

- Vers le tournant de notre ère, la statue d'Aristokléas fils de Philonikos est offerte

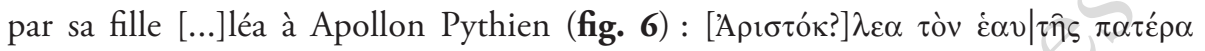

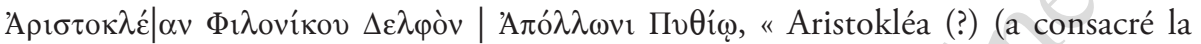
statue) de son père Aristokléas fils de Philonikos de Delphes à Apollon Pythien ${ }^{51}$. Un seul bloc (h. cons. 0,542 m; l. cons. 0,646 m; ép. 0,168 m) avec mortaises pour goujons et crampons au lit d'attente nous est parvenu de cette base qui devait être de grande taille. La personne honorée n'a pas d'enfant connu en dehors de cette fille; celle-ci aurait ainsi accompli un geste de piété filiale qui aurait pu être attendu de descendants mâles. Aristokléas a été bouleute sous N10 ( ca 30) et N22 (ca 20-10); il faut sans doute l'identifier avec l'archonte O1 (après 5-10 apr. J.-C.). Son père, Philonikos fils de Diodôros (I), a été peut-être archonte en M33 (nom restitué; ca 80-60), bouleute en M46 (milieu du i ${ }^{\text {er }}$ s.). Son oncle, Archias, a été bouleute en M14 (sans patronyme; ca 80-70). Son frère est sans doute le prêtre d'Apollon Diodôros fils de Philonikos, qui a été plusieurs fois bouleute (N18, N27, N41, N42, N43, N44, entre ca 30 et le début du I ${ }^{\text {r }}$ s. apr. J.-C.) et archonte (N40, N41 : ca 1 à 20 apr. J.-C.). Cette proche parenté n'est pas sans intérêt : le prêtre Diodôros est un des personnages qui incarnent le grand tournant politique du dernier tiers du I ${ }^{\text {er }} s^{52}$. Il est l'un des premiers à exercer plusieurs archontats, pratique inconnue pendant les quatre siècles précédents qui devient alors courante, marquant la concentration des fonctions politiques dans un cercle plus restreint. Il est également un des premiers Delphiens à se voir offrir une statue par ses concitoyens en tant qu'évergète du gymnase ${ }^{53}$. Il est enfin le proposant d'un décret en l'honneur d'un enseignant, daté du tournant de notre ère, dans lequel, parmi des considérants stéréotypés, se trouve

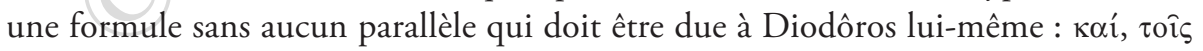

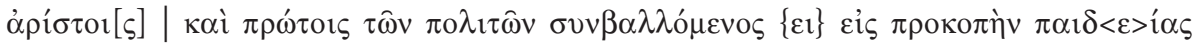

51. Inv. 3260. H. Ромтоw, "Ein delphisches Exemplar von "Kassanders Ehrentafel” und die delphischen Inschriften aus Bd. VIII d. Z. ", Hermes 41 (1906), p. 374-375. KYrIAKIDIs, $\Delta \varepsilon \lambda \varphi \omega \hat{\omega} v \pi \circ \lambda \iota \tau \varepsilon i ́ \alpha$, p. 429, no 35. Voir JACQUEMin, Offrandes, no 241.

52. Nous comptons rapidement revenir avec R. Bouchon sur le passage de l'époque hellénistique à l'époque impériale à Delphes.

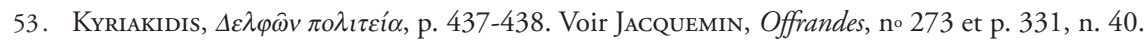




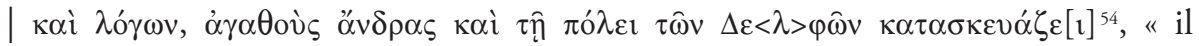
[l'honorandus] collabore avec les meilleurs et les premiers des citoyens au progrès de l'éducation et des belles-lettres et prépare ainsi des hommes de bien pour la cité de Delphes ${ }^{5}$ ». Cette formule montre un renouvellement marquant de la rhétorique civique, toujours démocratique jusque-là; le discours publiquement admis s'est mis en accord avec l'évolution des pratiques politiques: ce sont les " meilleurs et les premiers " des citoyens qui dominent la vie civique.

- Achevons avec un monument - celui de Diodôros fils de Dôrothéos - unique dans la documentation qui concerne les Delphiens et qui n'est peut-être pas à proprement parler une offrande, même si sa forme et sa consécration dans le sanctuaire devaient l'assimiler à ce type de monuments ${ }^{56}$. Il s'agit d'une construction constituée de plaques moulurées (h. min. $0,55 \mathrm{~m}$ ), sur lesquelles fut gravée, $c a$ 40-30, une collection de décrets honorifiques pour Diodôros - plus de onze textes émanant de différentes communautés : Lacédémone, koinon thessalien, Doriens de la métropole, Zakynthos, Thespies, Hyampolis, etc. La moulure des plaques de marbre semble dater de l'époque archäque; elles ont été ravalées partiellement et remployées pour la construction du monument de Diodôros, où elles ont été mises en œuvre avec des plaques de calcaire taillées pour l'occasion et qui tentaient de les imiter. Les plaques les plus grosses ont été découvertes au Sud du péribole, ce qui amène É. Bourguet à localiser hypothétiquement le monument de Diodôros dans la première partie de la " voie sacrée ". Il est donc possible, mais non certain, qu'il se soit agi d'une offrande à la divinité. Diodôros a été bouleute (M45: milieu du Ir s.), archonte (N15:ca 30-20), prêtre d'Apollon et bienfaiteur du gymnase. C'est l'un des cinq hiéromnémons envoyés auprès du césarien Kallistos de Cnide ${ }^{57}$. Son père, Dôrothéos fils de Diodôros, a été bouleute (M15) et archonte (M11) dans les années 80-70 ainsi que hosios. À la mort de Diodôros (fin du premier tiers du ir ${ }^{e r}$.), la cité de Delphes a voté un décret de consolation adressé à son fils Philoneikos : il s'agit d'un des premiers décrets connus pour un Delphien dans sa cités8. Diodôros fils de Dôrothéos est donc un alter ego du prêtre homonyme fils de Philonikos que nous avons déjà rencontré.

54. FD III 4, 59, 1. 7-9.

55. Trad. G. Colin légèrement modifiée.

56. Inv. 344+3320+4235+2833+3849+1286+4513+4090+4154. FD III 1, 487-496; H. Poмтоw (n. 19), p. 34-38, nos 55-60. Voir JaCQuemin, Offrandes, no 216; R. Bouchon, Les élites politiques de la cité de Delphes et $d u$ koinon des Thessaliens, cadre institutionnel, chronologique et pratiques familiales. Contribution à l'histoire politique et sociale de la Grèce centrale sous administration romaine, thèse, université de Lyon II (2005), p. 106-108 (présentation du personnage et datation du monument); KYRIAKIDIS, $\Delta \varepsilon \lambda \varphi \hat{\omega} v \pi \circ \lambda \iota \tau \varepsilon i \alpha$, p. 430, no $^{\circ} 36$.

57. CID IV, 130.

58. Sur ce décret, R. Bouchon (n. 56), p. 107-108 (commentaire) et p. 8 de l'annexe (réédition du texte). Sur le sens de cette évolution, voir Kyriakidis, $\Delta \varepsilon \lambda \varphi \hat{\omega} v \pi \circ \lambda \iota \tau \varepsilon i ́ \alpha$, p. 108. 


\section{L'ÉVOLUTION DES PRATIQUES DÉDICATOIRES DES DELPHIENS}

La trentaine d'offrandes delphiques dont j'ai présenté quelques exemples est inégalement répartie dans le temps (tabl. I).

\begin{tabular}{c|c} 
siècle & $\begin{array}{c}\text { nombre d'offrandes } \\
\text { conservées }\end{array}$ \\
\hline \hline $\mathrm{IV}^{\mathrm{e}}$ & 14 \\
\hline $\mathrm{III}^{\mathrm{e}}$ & 5 \\
\hline $\mathrm{II}^{\mathrm{e}}$ & 10 \\
\hline $\mathrm{I}^{\mathrm{er}}$ & 7 \\
\hline total & 36 \\
\hline \hline
\end{tabular}

Tabl. I - Répartition des offrandes par siècle.
Le niveau bas du III e $^{\mathrm{s}}$., au moment de la domination étolienne, est particulièrement notable, sans qu'il soit possible d'en avancer une explication vraiment satisfaisante. On ne connaît que cinq dédicaces pour tout le siècle; qui plus est, l'une d'entre elles pourrait s'avérer être une inscription funéraire. Dans l'hypothèse la plus favorable, c'est tout de même trois fois moins qu'au siècle précédent et deux fois moins qu'au siècle suivant. Les Étoliens occupent certes la première place dans le sanctuaire ${ }^{59}$, mais la cité de Delphes est formellement indépendante à cette époque et elle vote et fait graver ses décrets à un rythme soutenu, comme jamais au cours de son histoire. Le sanctuaire semble toujours très fréquenté et il serait hasardeux de conclure à une crise économique de ce seul critère, surtout au vu des lacunes inévitables dans cette documentation. Peut-être faudrait-il alors songer à une mauvaise conservation des monuments de cette époque, pour des raisons restant à expliquer. À cette exception près, la chronologie des offrandes privées suit l'évolution générale de l'épigraphie publique de la cité de Delphes, qui apparait au début du Ive s. Cette chronologie n'est pas spécifique : pour rester en Grèce centrale, à Gonnoi, par exemple, mais aussi Thespies, on constate une augmentation significative du nombre des dédicaces au IV $\mathrm{s}$. Le vide documentaire du demi-siècle qui suit la guerre mithridatique est valable lui dans tous les domaines de l'épigraphie delphique et s'explique sans doute par un appauvrissement marqué à l'échelle régionale puisque l'on constate un phénomène analogue, peut-être plus marqué encore, à Thespies ou à Chéronée ${ }^{60}$. Le creux dans la série des offrandes serait un témoignage direct des difficultés économiques des plus riches des Delphiens qui choisiraient pendant deux générations des formes de piété et de mise en scène de soi moins coûteuses.

La rupture la plus importante dans la série vient de l'apparition de l'offrande de statues de mortels vivants, qui très rapidement s'impose comme l'offrande monumentale

59. Voir la suggestion de MA, Statues, p. 223 et n. 156.

60. Voir Chr. Müller, «La procédure d'adoption des décrets en Béotie de la fin du III ${ }^{\text {e }}$ s. av. au I ${ }^{\text {er }}$ s. apr. J.-C. ", dans P. Fröhlich, Chr. Müller (éds), Citoyenneté et participation à la basse époque hellénistique. Actes de la table ronde des 22 et 23 mai 2004, Paris, BNF (2005), p. 106. 
par excellence. Si l'on met à part deux cas exceptionnels de la fin du IV ${ }^{\mathrm{e}} s$. et du $\mathrm{III}^{\mathrm{e}} s .{ }^{61}$, on constate que les familles les plus en vue de Delphes dédient communément de telles statues à partir du début du $\mathrm{II}^{\mathrm{e}}$ s., comme le fait la cité pour les statues honorifiques. Il y a ici une rencontre manifeste entre pratiques privées et pratiques publiques qui contribue à une reconfiguration religieuse des offrandes monumentales au profit de la figure d'Apollon Pythien ${ }^{62}$. Cette périodisation est, en Grèce centrale, spécifiquement delphique pour partie : à Thespies, les statues de proches sont courantes dès le IVe s., mais il est vrai qu'elles sont limitées au culte d'Artémis Ilithyie et qu'elles représentent sans doute la bénéficiaire de l'heureux accouchement. À Gonnoi, il ne semble pas que des particuliers aient pris l'habitude d'offrir des statues de mortels. Au contraire, de telles statues sont courantes à Athènes dès le milieu du IV s., à Orôpos dès le début du $\mathrm{III}^{\mathrm{e}} \mathrm{s}$., particulièrement dans les familles qui exercent d'importantes fonctions civiques $^{63}$. Au III $^{\mathrm{e}}$ s., la modestie des offrandes delphiques jure avec les monuments familiaux des élites étoliennes qui, sur deux colonnes ioniques et une architrave, supportent des groupes familiaux ${ }^{64}$. Les Delphiens ne rejoignent ce mouvement de consécration de monuments familiaux que lorsqu'il est quantitativement à son apogée à Athènes, Orôpos, Cnide ou Rhodes ${ }^{65}$. C'est encore l'exemple délien qui semble le plus proche: les offrandes de statues de proches débutent au $\mathrm{III}^{\mathrm{e}}$ s., mais l'essentiel de la série semble dater de la fin du $\mathrm{III}^{\mathrm{e}} \mathrm{s}$. et du début du $\mathrm{II}^{\mathrm{e}} \mathrm{s} .{ }^{66}$. Les Delphiens se mettent donc au diapason de cette mode "transcivique" au moment où elle est la plus forte, peut-être dans le contexte des retombées politiques et économiques (?) de l'expulsion des Étoliens. Le caractère en apparence plus riche des offrandes des $\mathrm{II}^{\mathrm{e}}-\mathrm{I}^{\mathrm{er}} s$. par rapport à celles des $\mathrm{IV}^{\mathrm{e}}-\mathrm{III}{ }^{\mathrm{e}} \mathrm{s}$. peut tout aussi bien s'expliquer par un enrichissement de l'élite économique de la cité que par une recherche accrue de munificence ou, plus simplement, par la baisse du coût de tels monuments, ce dernier phénomène semblant bien établi ${ }^{67}$.

61. Dédicace de la cité de Delphes à Oropos, fin du IV s. : D. Knoepfler, Décrets érétriens de proxénie et de citoyenneté, Eretria. Fouilles et recherches XI (2001), p. 388-389. Offrande de sortie de charge déjà évoquée plus haut, voir JACQUEmin, Offrandes, p. 45 et n. 60 ; no 253 ; Krriakidis, $\Delta \varepsilon \lambda \varphi \omega \hat{\omega} v \pi \circ \lambda ı \tau \varepsilon i ́ \alpha$, p. 420 , no 20 .

62. Voir la remarque de JacQuemin, Offrandes, p. 44.

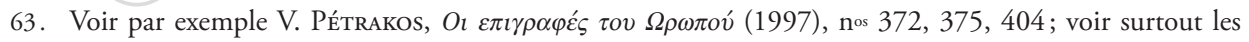
nos 405 et 426 (statues de prêtres d'Amphiaraos offertes par des membres de la famille).

64. JaCQuemin, Offrandes, nos 297, 298, 300, 299 (monuments à deux colonnes). Voir également le no 295 (banc de dix mètres surmonté de statues des membres de la famille). Voir aussi MA, Statues, p. 223-224.

65. Cadre général apud Ma, Statues, p. 196-202.

66. Pour Délos : IGXI 4, 1166-1189. Contrairement à Delphes, l'exercice de fonctions civiques est souvent l'occasion d'une offrande : IG XI 4, 1137-1142 (archontes); 1143-1147 (agoranomes et astynomes); 1151-1154 (gymnasiarques), ceci sans compter les dédicaces agonistiques.

67. Voir MA, Statues, p. 245. 


\section{Iv. PARTICIPATION À LA VIE PUBLIQUE ET NOTABILITÉ POLITIQUE}

Pour comprendre la place qu'occupe le groupe de ces dédicants dans la vie politique de la cité delphique, il convient de saisir la signification des fonctions exercées en précisant le régime de participation au sein duquel se déroulent ces " carrières".

Au IVe et au III s. , le patronyme des Delphiens n'est mentionné qu'exceptionnellement, ce qui explique certaines hésitations dans les rapprochements prosopographiques envisageables. Ce n'est donc qu'au II $s$. que l'étude des familles, et non plus seulement des individus, est pleinement possible, lorsque l'on dispose de patronymes et de la possibilité de croiser les renseignements fournis par les actes d'affranchissements et ceux que donnent les décrets de la cité. De plus, la documentation ne concerne pas les mêmes archai suivant les époques. La seule série continue dans le temps est celle des titulaires de l'archontat éponyme; elle constitue donc ici un indicateur privilégié. On connait, du début du IV $\mathrm{s}$. à la fin du Ire s. plus de $90 \%$ des titulaires de cette magistrature et, pour certaines périodes, ce taux est presque de $100 \%$. À deux moments de l'histoire de la communauté, on peut croiser plusieurs séries partielles qui permettent une réelle étude du personnel politique en synchronie.

Pour les années 346-312, tous les archontes ou presque sont connus, ainsi que $60 \%$ des prytanes (un collège de huit magistrats annuels chargés de la garde des biens d'Apollon) pendant 26 années et, pour une décennie, la moitié des bouleutes (quinze membres en fonction pour un semestre, donc trente par an). Sont également connus à cette époque des noms de Delphiens désignés par leur communauté pour siéger dans les instances amphictioniques : ceux de la moitié ou presque des hiéromnémons annuels, là encore pendant une vingtaine d'années, ceux des naopes, dont la charge n'est pas limitée dans le temps. On dispose pour la même époque de quelques mentions du nombre des votants à l'Assemblée du peuple et l'on peut en conclure qu'il pouvait y avoir de 400 à 600 citoyens qui y siégeaient. L'étude détaillée du recrutement de ces magistratures permet d'infirmer l'opinio communis qui fait de la Delphes du ive s. une cité oligarchique ${ }^{68}$. L'itération des fonctions de prytane ou de hiéromnémon est exceptionnelle et ne concerne au maximum que $10 \%$ des titulaires - en fait beaucoup moins -, généralement pour un seul mandat supplémentaire. Il en va de même pour les fonctions de bouleute : s'il n'est pas possible de déterminer un nombre maximum de mandats exercés au cours d'une vie, il est habituel de ne pas siéger au Conseil plus d'une fois par décennie et les exceptions sont rares (15\% au maximum pour un second mandat). Ce sont donc quelque deux

68. Historiographie de ces jugements : Ph. Gauthier (n. 7), p. 110-111; Kyriakidis, $\Delta \varepsilon \lambda \varphi \hat{\omega} v \pi o \lambda \iota \tau \varepsilon i ́ \alpha$, p. 66-70. Malgré la démonstration de Ph. Gauthier sur la nature démocratique des institutions delphiques au $\mathrm{II}^{\mathrm{e}} \mathrm{s}$. - conclusion que je crois pouvoir étendre à l'ensemble du régime de participation et ce dès le IVe s. -, voir par ex. A. CASSAYRE, La Justice dans les cités grecques. De la formation des royaumes hellénistiques au legs d'Attale (2010), p. 351, qui témoigne de la persistance de l'opinio communis. 
cents citoyens différents qui pouvaient devenir bouleutes tous les huit ans et, en une quinzaine d'années, c'est une centaine de Delphiens qui avaient pu être prytanes. On peut en conclure qu'une part importante de la population civique, de l'ordre du quart ou du tiers, avait accès à une fonction politique au moins une fois dans sa vie. Ce large accès aux archai n'empêchait pas que certains exerçassent un grand nombre de fonctions différentes au cours d'une vie - nous en avons quelques indices -, mais il ne leur était pas possible de se maintenir durablement dans la même fonction.

L'autre période pour laquelle l'analyse est possible est le $\mathrm{II}^{\mathrm{e}} \mathrm{s}$., particulièrement sa première moitié. L'image donnée par la documentation y est différente, mais il est vrai que ce ne sont plus les mêmes fonctions qui sont connues. L'archontat n'est toujours exercé qu'une fois au cours d'une vie. On dispose désormais de tous les noms des titulaires des deux prêtrises viagères d'Apollon Pythien. Le nom de presque tous les bouleutes nous est également parvenu; cependant, sous ce terme de bouleute ne sont plus désignés les quinze membres du Conseil semestriel, mais trois magistrats qui forment sans doute la commission permanente d'un conseil plus large dont les membres sont peut-être appelés probouloi $i^{69}$; les bouleutes du $\mathrm{II}^{\mathrm{e}} \mathrm{s}$. ne peuvent donc être comparés à ceux du IV $s$. Les fonctions paraissent un peu plus concentrées : le nombre moyen de mandats de bouleute est de deux au cours d'une vie; alors que la moitié des bouleutes ne l'ont été qu'une ou deux fois, l'autre moitié exerce de deux à six mandats, l'archontat (37\% de ceux qui ont été bouleutes ont été aussi archontes), parfois la prêtrise d'Apollon Pythien (6,5\% de ceux qui ont été bouleutes achèvent leur "carrière " en exerçant ce sacerdoce viager exclusif, au $\mathrm{II}^{\mathrm{e}}$ s., de toute autre fonction civique importante). La quasi-totalité des intervenants à l'Assemblée et des ambassadeurs connus, mais aussi presque tous les prêtres d'Apollon et les archontes ont déjà été bouleutes ou, plus rarement, le sont - pour les archontes dans la suite de leur carrière. Si l'accès aux fonctions politiques n'est donc pas également réparti et que la fréquence de l'exercice des archai est très inégale, il serait néanmoins imprudent de parler d'une oligarchisation. 478 citoyens de Delphes nous sont connus grâce aux actes d'affranchissement entre 170 et 144 (ce nombre peut comprendre des adolescents qui ne sont pas parvenus à l'âge des responsabilités politiques); 167 ont été bouleutes ou archontes au cours de leur vie, soit 35\% d'entre eux. Il n'est pas possible d'en déduire que les autres n'ont exercé aucune fonction : on ne sait rien du recrutement des prytanes ou des membres d'un conseil plus large - deux institutions qui permettaient justement, au IVe s., à un grand nombre de citoyens d'accéder à un niveau de participation politique supérieur à la seule présence à l'Assemblée. On ne connaît pas non plus la part de la population civique totale que représentent ces Delphiens mentionnés dans les actes d'affranchissement, même si à cette époque on possède sans doute la quasi-totalité de ces documents.

69. Kyriakidis, $\Delta \varepsilon \lambda \varphi \hat{\omega} v \pi o \lambda \imath \tau \varepsilon i ́ \alpha$, p. 121-123, contra Ph. Gauthier (n. 7), p. 123-124. 
Malgré les nuances entre les deux tableaux que je viens de dresser, il existe un certain nombre de points communs entre ces deux périodes : l'exercice des responsabilités politiques n'est jamais le fait d'une étroite minorité, mais elle n'est jamais non plus égalitaire. Certains exercent continûment ou presque des fonctions civiques, alors que d'autres ne le font que rarement et d'autres encore jamais. À la suite de plusieurs participants du colloque Les élites et leurs facettes ${ }^{70}$, j'utilise pour qualifier les deux premiers groupes le terme de "notabilité politique ", dans le sens wébérien du terme : des citoyens qui grâce à leur place dans les rapports économiques peuvent bénéficier d'un temps libre qu'ils choisissent d'utiliser pour assurer bénévolement le fonctionnement de leur communauté et qui, pour ce faire, jouissent de la confiance de leurs concitoyens, sans pour autant constituer une classe distincte de la sociétép ${ }^{1}$. Je ne crois pas que l'on puisse, concernant le personnel politique de Delphes, parler d' «élite", c'est-à-dire, pour reprendre la définition d'É. Perrin-Saminadayar, d'un "groupe de gens qui sortent du lot commun, reconnus comme tels et désireux de l'être, dont les comportements s'individualisent et sont caractéristiques de sa prétendue supérioritép ${ }^{2}$ ", définition qui me paraît cependant correspondre, comme je l'ai montré plus haut, aux dédicants des offrandes monumentales. Un argument peut être avancé pour étayer mon point de vue, valable pour l'une et l'autre périodes évoquées : tous les individus considérés exercent les mêmes fonctions (à l'exception des fonctions viagères), l'inégalité étant dans la fréquence, non dans la nature des fonctions exercées. Ainsi, il n'y a pas de yrai monopole d'un groupe sur une fonction pour autant que l'on puisse en juger: les membres de ce que j'ai appelé le "premier cercle de participation " $^{73}$, caractérisé par plusieurs bouleutats, l'exercice de l'archontat ou de la prêtrise d'Apollon, qui prennent le plus part au fonctionnement des institutions au cours de leur vie, exercent des fonctions différentes tout au long de leurs «carrières ». Par exemple, le "champion" absolu de l'itération du bouleutat au II ${ }^{e}$ s., Hagiôn fils d'Éképhylos, qui a été aussi archonte et prêtre d'Apollon, a été six fois bouleute en trente ans, soit "seulement" dans un collège sur dix ${ }^{74}$.

70. M. Cébeillac-Gervasoni, L. Lamoine (éds), Les élites et leurs facettes. Les élites locales dans le monde hellénistique et romain (2003). En dernier lieu : L. CAPdetrey, Y. LAFOND (éds), La cité et ses élites. Pratiques de domination et représentations du contrôle social dans les cités grecques (VIII s. a. C.-Fer s. p. C.), Études 25 (2010).

71. I. Savalli-Lestrade, "Remarques sur les élites dans les poleis hellénistiques ", dans M. CébeillacGervasoni, L. Lamoine (éds) [supra], p. 51 et n. 2.

72. É. Perrin-Saminadayar, "Des élites intellectuelles à Athènes à l'époque hellénistique? Non, des notables ", dans M. Cébeillac-Gervasoni, L. Lamoine (éds) [n. 70], p. 384.

73. KyriaKidis, $\Delta \varepsilon \lambda \varphi \hat{\omega} v \pi 0 \lambda \imath \tau \varepsilon i ́ \alpha$, p. 291, 353.

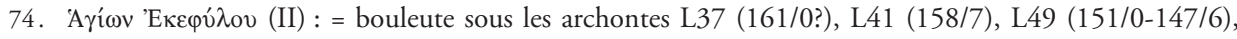
L55 (151/0-147/6), L57 (142/1 ou 141/0), L65 (gr.) (134/3? ou 130/29?), archonte L63 (130/29? ou 134/3), prêtre d'Apollon entre 121 et 108, pendant au moins six années archontales. Références chez G. Daux, Chronologie delphique (1943), sous ces archontats. 
Superposons les deux images que je viens de décrire : celle, d'une part, d'une élite socio-économique - les dédicants d'offrandes monumentales - et, d'autre part, celle de la notabilité politique delphique, un groupe large et différencié, et revenons aux pratiques dédicatoires des Delphiens pour voir si leur évolution dans le temps peut refléter un changement sociopolitique dans la cité.

\section{v. DÉDICANTS ET MAGISTRATS}

Au IVe s., parmi les quatorze offrandes de Delphiens dont nous disposons (dont trois reliefs), on peut isoler neuf noms de dédicants de sexe masculin. Dans cinq de ces cas, le dédicant n'est pas attesté dans le personnel politique. On peut certes incriminer alors les vides de la documentation : nous ne connaissons qu'une partie des bouleutes, des prytanes et des hiéromnémons. Plus particulièrement, le nom d'aucun des prêtres d'Apollon de cette période ne nous est parvenu. Cet argument du silence ne vaut cependant pas pour les archontes: or, nous ne possédons aucune offrande attribuable à un ancien ou futur archonte au Ive $s$. Les offrandes des Delphiens sont relativement modestes : il s'agit souvent de piliers pour statuettes. On ne connait pas exactement la nature des agalmata qu'elles supportaient : vraisemblablement une représentation de la divinité honorée, peut-être des trépieds votifs ou tout autre objet approprié ${ }^{75}$. Le formulaire de la dédicace est sommaire et se contente de mentionner le nom du dédicant, celui de la divinité et la formule de consécration, comme il est courant dans la région. Il est frappant que l'élite économique de la cité se distingue par la modestie de ses programmes, alors même que, dans le principal sanctuaire civique, elle peut voir le monument de Daochos exposer sur huit générations les gloires d'une des principales familles thessaliennes ${ }^{76}$. Cest sans doute un témoignage du caractère limité des moyens des notables de Delphes, mais peut-être aussi d'une certaine retenue due à une pression sociale réprouvant les marques de distinction. Il ne semble pas y avoir eu alors, du moins en l'état actuel de la documentation, certes assez lacunaire, de corrélation entre offrandes de prestige et carrière politique de premier plan. Le seul contre-exemple serait l'offrande d'Asôpodôros, s'il s'agit bien de l'Asôpodôros fils de Sakedallos : une grande base de statue signée par un bronzier de renom, offerte par un personnage qui a été naope, fonction exercée par certains personnages de premier plan dans la cité (Étymôndas par exemple, dont on ne connaît pas d'offrande) ${ }^{77}$. À deux occasions peut-être, ces dédicaces

75. Voir par exemples les différentes possibilités recensées dans le catalogue de K. KIssAs (n. 2), et P. Amandry, «Trépieds de Delphes et du Péloponnèse », BCH 111 (1987), p. 79-131.

76. Voir en dernier lieu : A. Jacquemin, D. Laroche, " Le monument de Daochos ou le trésor des Thessaliens ", BCH 125 (2001), p. 305-332.

77. Pour un essai de reconstitution de la « carrière » de ce personnage, voir KYRIAKIDIs, $\Delta \varepsilon \lambda \varphi \hat{\omega} v \pi o \lambda \iota \tau \varepsilon i ́ \alpha$, p. 286. 
mentionnent l'exercice d'un sacerdoce. L'une d'elles évoque l'accomplissement d'une prêtrise d'Ilithyie; cette catégorie des offrandes de sortie de charge est notablement rare à Delphes par rapport à ce que l'on peut observer à Gonnoi ou à Thespies pour les mêmes divinités à la même époque.

Au III e s., le nombre d'offrandes conservées est moindre et s'adresse à un cercle plus restreint de divinités. Cette période semble voir se perpétuer la situation qui prévalait antérieurement. Sur les cinq dédicants de sexe masculin, quatre n’ont apparemment été ni bouleutes (ce qui n'est pas contraignant) ni archontes, ce qui reste un bon indicateur. Le constat est le même pour les pères des dédicants, lorsque leur nom est connu. Le seul dédicant qui peut avoir été archonte ou bouleute est fils d'un citoyen dont le nom est également attesté parmi les titulaires de ces fonctions. La seule vraie nouveauté, on l'a vu, est l'apparition d'offrandes de statues de mortels, parents, amis ou bienfaiteurs, mais ce dernier cas est encore exceptionnel : c'est plusieurs décennies après les Athéniens que les Delphiens suivent timidement l'exemple des grandes familles qui, à la fin du rve s. et au début du III e, dédient des statues d'arrhéphores, de prêtresses et de prêtres sur l'Acropole ${ }^{78}$.

Au début du II ${ }^{e}$ s., si la nature des offrandes monumentales change soudainement, il en va de même de leur contexte social. Ce sont désormais majoritairement des statues représentant des mortels (sur les dix offrandes connues, six consistent en des statues de proches parents $)^{79}$ et c'est justement à partir de cette date que leurs dédicants appartiennent presque tous à la plus haute notabilité politique de la cité. Pour reprendre le critère de l'archontat, six offrandes sur dix sont faites par un ancien ou futur archonte, par une épouse d'archonte ou représentent un archonte. Une offrande supplémentaire est le fait d'un citoyen du même milieu, deux autres offrandes ont été faites par des bouleutes. Une seule offrande émane d'un Delphien qui n'apparait pas dans le personnel politique. Les dédicants sont presque toujours issus de ce que j’ai appelé le "premier cercle de la participation ". Dès lors, il me semble que ces monuments sont revêtus d'une nouvelle fonction dans la société delphique. Ils affirment l'excellence sociale des plus grands notables, mettent en scène devant la communauté l'unité du groupe familial - soudé par l'affection - garante de la continuité des vertus de ses membres et célèbrent ses réussites, comme les victoires au concours. L'étroitesse du milieu qui met en œuvre ces stratégies est

78. Voir R. von Den Hoff, "Tradition and Innovation: Portraits and Dedications on the Early Hellenistic Akropolis ", dans O. Palagia, S. V. Tracy (éds), The Macedonians in Athens, 322-229 B. C.: Proceedings of an International Conference Held at the University of Athens, May 24-26, 2001 (2003), p. 173-185. De manière générale, sur les offrandes "familiales " des périodes les plus anciennes, voir Chr. LöHR, Griechische Familienweihungen: Untersuchungen einer Repräsentationsform von ihren Anfängen bis zum Ende des 4. Jhs. v. Chr., Internationale Archäologie 54 (2000).

79. Je ne compte pas l'offrande d'un certain Darikos, qui ne répond pas au critère de monumentalité et d'ostentation; de plus, le dédicant est sans doute un affranchi. Voir JaCQUEMIN, Offrandes, no 650. 
frappante : sur les dix offrandes connues pour le siècle, huit sont le fait de trois familles dont tous les membres - ou presque - exercent des fonctions de premier plan, et, parmi celles-ci, plus spécifiquement la prêtrise d'Apollon Pythien. Sur ces huit offrandes, quatre sont attribuables à la seule famille de Praxias et Praxô.

En rupture avec les deux siècles précédents, les offrandes monumentales des Delphiens au II ${ }^{e} s$. nous donnent l'image très cohérente de quelques familles qui s'efforcent d'occuper une place centrale dans la vie politique et sociale de la cité et qui se mettent en scène en tant que telles à travers des groupes statuaires offerts à la principale divinité de la cité. Il est particulièrement intéressant que cette ostentation sociale au service du prestige et de la cohérence de groupes familiaux se déroule dans une cité régie par des institutions démocratiques qui font la part belle aux pouvoirs de l'Assemblée et où, aux côtés de ces grands notables, une part significative de la population civique accède aux fonctions politiques. Les pratiques sociales semblent ici ouvrir la voie à une évolution politique et institutionnelle qui est largement postérieure.

Les sept monuments conservés pour le $\mathrm{I}^{\mathrm{er}} \mathrm{s}$. poursuivent les traditions du II ${ }^{\mathrm{e}}$., notamment par la mise en scène des liens qui unissent les membres des principales familles de la cité. Le monument de Diodôros fils de Dôrothéos se distingue par son ambition. C'est dans le sanctuaire et sans doute par l'entremise d'une consécration que ce grand notable des années 40 a décidé de donner à voir à ses concitoyens et à tous les pèlerins la collection des décrets honorifiques rendus en son honneur par diverses cités et confédérations. C'est dans ce contexte social et culturel que se développent, à Delphes, les innovations des débuts de l'époque impériale : l'offrande de statues de Delphiens honorés par leurs concitoyens, qui est une nouveauté de cette période, à laquelle font écho dans le champ idéologique et institutionnel le changement dans un sens oligarchique de la rhétorique civique, la possibilité d'itérer l'archontat ou de cumuler le bouleutat et la prêtrise d'Apollon Pythien, tous éléments qui contribuent à augmenter la distance sociale entre ce que l'on peut désormais appeler une élite sociopolitique et le reste des citoyens ${ }^{80}$.

\section{CONCLUSION : DEUX RÉGIMES SUCCESSIFS DE L'OFFRANDE DANS UNE SOCIÉTÉ EN MUTATION}

$\mathrm{Du} \mathrm{IV}^{\mathrm{e}}$ au I $\mathrm{I}^{\mathrm{er}} \mathrm{s}$. avant notre ère, la société delphique a donc connu deux régimes successifs de l'offrande monumentale. Lors d'une première période, il ne semble y avoir eu aucune corrélation forte entre dédicace de ce type d'offrandes et exercice du pouvoir politique

80. Sur les monuments funéraires de l'époque impériale, voir par exemple J.-Fr. Bommelaer, Guide de Delphes. Le site (1991), p. 221 (hérôon dit « de Blum ») ou FD IV 6, no 30, p. 109-123 (sarcophage dit « de Méléagre »). 
dans la cité. Si cela a été le cas, l'état de la documentation ne nous permet pas de l'observer. C'est alors même que la nature démocratique des institutions n'est plus contestable que le sommet de la notabilité politique de Delphes décide de se mettre en scène à travers des monuments qui exaltent l'unité de la famille et l'excellence de ses membres. Le fait que des modèles de ce comportement existaient à Delphes même dans le sanctuaire d'Apollon Pythien dès le rve s. ne marque pas à proprement parler un retard des Delphiens, mais plutôt l'indice que l'évolution sociopolitique de la cité du Parnasse a suivi un rythme propre et que ses citoyens ne se sont emparés d'une pratique bien établie que lorsqu'ils en ont ressenti socialement le besoin - ou lorsqu'ils en ont eu les moyens. Le mouvement de concentration de l'exercice du pouvoir politique dans un milieu étroit lors de la seconde moitié du $\mathrm{I}^{\text {er }}$ s. a pu s'appuyer sur des pratiques de distinction sociale déjà courantes dans la société delphique. L'« aristocratisation » de certaines pratiques sociales aurait ainsi précédé de plus d'un siècle celle de la structure politique et idéologique de la cité ${ }^{81}$.

Malgré les lacunes de la documentation, qui ne doivent pas être perdues de vue, surtout pour le $\mathrm{IV}^{\mathrm{e}}$ et le $\mathrm{III}^{\mathrm{e}} \mathrm{s}$., la démarche consistant à mettre en regard " carrières " politiques et pratiques affirmant une supériorité sociale me parait avoir montré sa fécondité. Elle permet ainsi de restituer les stratégies mises en ouvre par les acteurs pour assurer leur prééminence dans leur communauté. Cette démarche a déjà été utilisée par différents chercheurs pour l'histoire grecque archaïque, classique ou même impériale ${ }^{82}$, mais elle n'avait été que très ponctuellement appliquée aux notables de l'époque hellénistique jusqu'au récent ouvrage de J. Ma qui est appelé à constituer un jalon important dans l'étude de ces pratiques ${ }^{83}$. Sa systématisation et son élargissement à toutes les formes d'ostentation pourraient peut-être contribuer à mieux éclairer l'histoire des milieux favorisés de cette période, hors de l'exemple des plus grandes cités qui est généralement le plus sollicité. Ainsi, le témoignage de la cité de Delphes nous donne un accès précieux au monde des cités petites et moyennes qui furent si nombreuses et qui sont, pour la plupart, si mal documentées.

81. Pour une vision d'ensemble du processus d' «aristocratisation " des élites des cités grecques à la fin de l'époque hellénistique, voir P. HAMON, "Élites dirigeantes et processus d'aristocratisation à l'époque hellénistique", dans H.-L. Fernoux, Chr. STeIn (éds), Aristocratie antique. Modèles et exemplarité sociale (2007), p. 79-100.

82. Voir par exemple A. Duplouy, Le prestige des élites. Recherches sur les modes de reconnaissance sociale en Grèce entre les Xe et Ve siècles avant J.-C. (2006), ou, du même auteur, "Du voisinage à la sphère internationale : cercles de collectivité et niveaux d'énonciation des modes de reconnaissance sociale dans l'Athènes classique ", dans J.-Chr. Couvenhes, S. Milanezi (éds), Individus, groupes et politique à Athènes de Solon à Mithridate, Actes du colloque international, Tours, 7 et 8 mars 2005 (2007), p. 35-55; pour l'époque impériale, voir l'introduction et plusieurs contributions du colloque publié par A. Heller, A.-V. Pont (éds), Patrie d'origine et patries électives. Les citoyennetés multiples dans le monde grec d'époque romaine, Scripta Antiqua 40 (2012).

83. MA, Statues. 


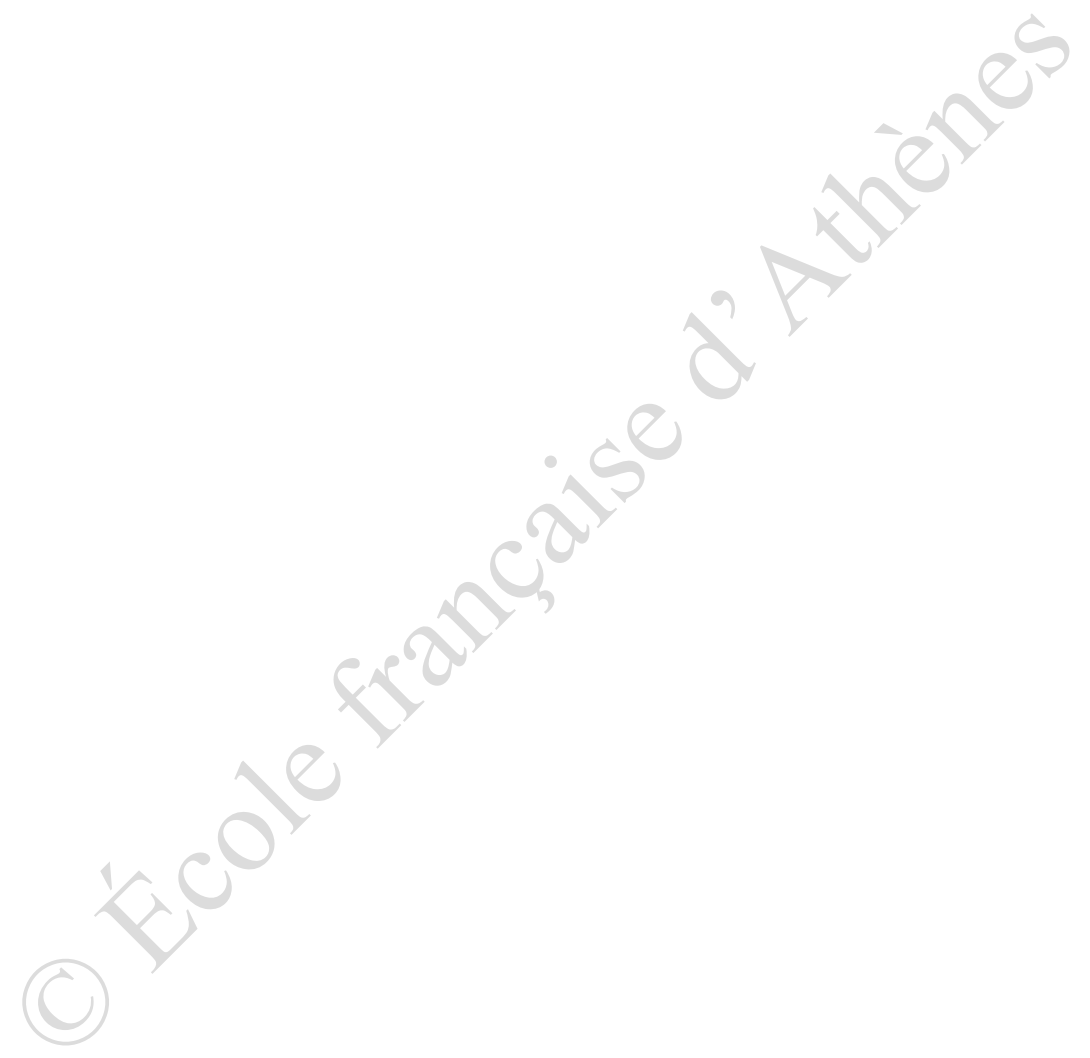

